TECHNISCHE UNIVERSITÄT MÜNCHEN INSTITUT FÜR INFORMATIK

Completeness Results for Generalized Communication-free Petri Nets with Arbitrary Edge Multiplicities

Ernst W. Mayr and J eremias Weihmann

TUM-I1335 


\title{
Completeness Results for Generalized Communication-free Petri Nets with Arbitrary Edge Multiplicities
}

\author{
Ernst W. Mayr* Jeremias Weihmann*
}

July 19, 2013

\begin{abstract}
We investigate gcf-Petri nets, a generalization of communication-free Petri nets allowing arbitrary edge multiplicities, and characterized by the sole restriction that each transition has at most one incoming edge. We use canonical firing sequences with nice properties for gcf-PNs to show that the RecLFS, (zero-)reachability, covering, and boundedness problems of gcf-PNs are in PSPACE. By showing, how PSPACE-Turing machines can be simulated by gss-PNs, a subclass of gcf-PNs where additionally all transitions have at most one outgoing edge, we ultimately prove the PSPACE-completess of these problems for gss/gcf-PNs. Then, we show PSPACE-hardness as well as a doubly exponential space bound for the containment, and equivalence problems of gss/gcf-PNs. Last, we consider a new natural generalization of context-free commutative grammars. Our results for gcf-PNs imply PSPACE-completeness for its uniform word problem.
\end{abstract}

\section{Introduction}

In [17, Mayr proposed a non-primitive recursive algorithm for the general Petri net reachability problem, thus proving its decidability. For many restricted Petri net classes, a better complexity of the reachability problem can be shown. However, the nets of most Petri net classes for which the complexity of the reachability problem could be refined are subject to the restriction that all edges from places to transitions have multiplicity one. Well known examples of such nets with NP-complete reachability problems are communication-free Petri nets (cf-PNs/BPP-PNs), [4, 23], conflict-free Petri nets [8, 9] and normal as well as sinkless Petri nets [9] (for the latter two, the promise problem variation of the reachability problem was considered). Remarkable examples for Petri net classes with general edge multiplicities and matching lower and upper bounds for the reachability problem are single-path Petri nets [7] (PSPACE-complete) and reversible Petri nets [18 (EXPSPACE-complete). For a more comprehensive overview, the reader is referred to [5].

Our ultimate goal is to gain insight into how general edge multiplicities influence the complexity of the reachability problem and several other classical problems. In this paper, we investigate a generalization of communication-free Petri nets. A cf-PN is a Petri net such that each transition has exactly one input place, connected by an edge with multiplicity one. Cf-PNs are closely related to Basic Parallel Processes defined in [1, 2, as well as to context-free (commutative) grammars 5, 11. We call our generalization generalized communication-free Petri nets (gcf-PNs). The nets of this class are characterized by a single topological constraint, namely, that each transition has at most one input place, connected by an edge with arbitrary multiplicity.

For cf-PNs, tight bounds for the reachability problem are known. Esparza [4] showed NPcompleteness while Yen [23] gave an alternative proof for NP-membership, based on canonical firing sequences. Both proofs (implicitly) rely on the fact that the RecLFS problem (recognize legal firing sequence, see [22]) is decidable in polynomial time due to a very easily checkable

\footnotetext{
*Institut für Informatik, Technische Universität München, D-85748 Garching, Germany, \{mayr,weihmann\}@in.tum.de
} 
criterion. (The problem RecLFS asks if a given Parikh vector is enabled at some given marking.) For gcf-PNs, no such criterion exists (under the assumption $\mathrm{P} \neq \mathrm{PSPACE}$ ) since the problem is PSPACE-complete as shown in Section 4.

In Section 3, we show PSPACE-hardness for the RecLFS, the reachability, the covering, and the boundedness problems of generalized S-Sytems (gss-PNs) which are a subclass of gcf-PNs where each transition has at most one incoming and at most one outgoing edge, each with arbitrary edge multiplicity. This is interesting because almost all the problems considered in this paper have very low complexity for S-Systems (e.g., they are always bounded, the reachability problem is decidable in polynomial time [6], etc.). Furthermore, the covering, and the boundedness problems of cf-PNs are known to be NP-complete, and linear time (on RAMs), respectively [16].

In Section 4 we derive canonical permutations of firing sequences of gcf-PNs, and use them to show PSPACE-completeness for the RecLFS, the reachability, and the covering problems of gcf-PNs.

In Section 5 , we show the existence of canonical firing sequences that have stronger properties than the firing sequences obtained by canonical permutations. These canonical firing sequences resemble those given in 23 for cf-PNs. We use them to show PSPACE-completeness for the boundedness problem of gcf-PNs, and that the equivalence and containment problems of gcf-PNs are PSPACE-hard as well as decidable in doubly exponential space.

In Section 66 we consider exponent-sensitive commutative grammars, a new natural generalization of context-free commutative grammars [11, which allow productions to substitute many occurrences of a single variable. We use our results for gcf-PNs to show that the uniform word problem of this class is PSPACE-complete.

\section{Preliminaries}

$\mathbb{Z}, \mathbb{N}_{0}$, and $\mathbb{N}$ denote the set of all integers, all nonnegative integers, and all positive integers, respectively, while $[a, b]=\{a, a+1, \ldots, b\} \varsubsetneqq \mathbb{Z}$, and $[k]=[1, k] \varsubsetneqq \mathbb{N}$. For two vectors $u, v \in \mathbb{Z}^{k}$, we write $u \geq v$ if $u_{i} \geq v_{i}$ for all $i \in[k]$, and $u>v$ if $u \geq v$ and $u_{i}>v_{i}$ for some $i \in[k]$. When $k$ is understood, $\vec{a}$ denotes, for a number $a \in \mathbb{Z}$, the $k$-dimensional vector with $\vec{a}_{i}=a$ for all $i \in[k]$.

A Petri net $N$ is a 3-tuple $(P, T, F)$ where $P$ is a finite set of $n$ places, $T$ is a finite set of $m$ transitions with $P \cap T=\emptyset$, and $F: P \times T \cup T \times P \rightarrow \mathbb{N}_{0}$ is a flow function. Throughout this paper, $n$ and $m$ will always refer to the number of places resp. transitions of the Petri net under consideration, and $W=\max \{F(p, t), F(t, p) \mid p \in P, t \in T\}$ to the largest value of its flow function. Usually, we assume an arbitrary but fixed order on $P$ and $T$, respectively. With respect to this order on $P$, we can consider an $n$-dimensional vector $v$ as a function of $P$, and, abusing the notation, write $v(p)$ for the entry of $v$ corresponding to place $p$. Analogously, we write $v(t)$ in context of an $m$-dimensional vector and a transition $t$.

A marking $\mu$ (of $N$ ) is a vector of $\mathbb{N}_{0}^{n}$. A pair $\left(N, \mu^{(0)}\right)$ such that $\mu^{(0)}$ is a marking of $N$ is called a marked Petri net, and $\mu^{(0)}$ is called its initial marking. We will omit the term "marked" if the presence of a certain initial marking is clear from the context.

For a transition $t \in T, \bullet^{\bullet} t\left(t^{\bullet}\right.$, resp.) is the preset (postset, resp.) of $t$ and denotes the set of all places $p$ such that $F(p, t)>0\left(F(t, p)>0\right.$, resp.). Analogously, the sets ${ }^{\bullet} p$ and $p^{\bullet}$ of transitions are defined for the places $p \in P$. A Petri net $(P, T, F)$ is a generalized communication-free Petri net (gcf-PN) if $|\bullet t| \leq 1$ for all $t \in T$. A gcf-PN is a generalized S-System Petri net (gss-PN) if additionally $\left|t^{\bullet}\right| \leq 1$ for all $t \in T$.

A Petri net naturally corresponds to a directed bipartite graph with edges from $P$ to $T$ and vice versa such that there is an edge from $p \in P$ to $t \in T$ (from $t$ to $p$, resp.) labelled with $w$ if $0<F(p, t)=w$ (if $0<F(t, p)=w$, resp.). The label of an edge is called its multiplicity. If a Petri net is visualized, places are usually drawn as circles and transitions as bars. If the Petri net is marked by $\mu$, then, for each place $p$, the circle corresponding to $p$ contains $\mu(p)$ so called tokens.

For a Petri net $N=(P, T, F)$ and a marking $\mu$ of $N$, a transition $t \in T$ can be applied at $\mu$ producing a vector $\mu^{\prime} \in \mathbb{Z}^{n}$ with $\mu^{\prime}(p)=\mu(p)-F(p, t)+F(t, p)$ for all $p \in P$. The transition $t$ is enabled at $\mu$ or in $(N, \mu)$ if $\mu(p) \geq F(p, t)$ for all $p \in P$. We say that $t$ is fired at marking $\mu$ if $t$ 
is enabled and applied at $\mu$. If $t$ is fired at $\mu$, then the resulting vector $\mu^{\prime}$ is a marking, and we write $\mu \stackrel{t}{\rightarrow} \mu^{\prime}$. Intuitively, if a transition is fired, it first removes $F(p, t)$ tokens from $p$ and then adds $F(t, p)$ tokens to $p$.

An element $\sigma$ of $T^{*}$ is called a transition sequence, and $|\sigma|$ denotes its length. For the empty transition sequence $\sigma=()$, we define $\mu \stackrel{\sigma}{\rightarrow} \mu$. For a nonempty transition sequence $\sigma=t_{1} \cdots, t_{k}$, $t_{i} \in T$, we write $\mu^{(0)} \stackrel{\sigma}{\rightarrow} \mu^{(k)}$ if there are markings $\mu^{(1)}, \ldots, \mu^{(k-1)}$ such that $\mu^{(0)} \stackrel{t_{1}}{\longrightarrow} \mu^{(1)} \stackrel{t_{2}}{\rightarrow}$ $\mu^{(2)} \ldots \stackrel{t_{k}}{\longrightarrow} \mu^{(k)}$. We write $\sigma_{(i, j)}$ for the subsequence $\sigma_{i} \cdot \sigma_{i+1} \cdots \sigma_{j}$, and $\sigma_{(i)}$ for the prefix of length $i$ of $\sigma$, i.e., $\sigma_{(i)}=\sigma_{(1, i)}$.

A Parikh vector $\Phi$, also known as firing count vector, is simply an element of $\mathbb{N}_{0}^{m}$. The Parikh map $\Psi: T^{*} \rightarrow \mathbb{N}_{0}^{m}$ maps each transition sequence $\sigma$ to its Parikh image $\Psi(\sigma)$ where $\Psi(\sigma)(t)=k$ for a transition $t$ if $t$ appears exactly $k$ times in $\sigma$. Note that each Parikh vector $\Phi$ is the Parikh image of some transition sequence. Furthermore, we write $t \in \Phi$ if $\Phi(t)>0$, and $t \in \sigma$ if $t \in \Psi(\sigma)$. For a transition sequence $\sigma \in T^{*}$, we define $\bullet^{\circ}=\bigcup_{t \in \sigma} \bullet$. $\Psi_{\text {first }}(\sigma)$ is the Parikh vector such that, for all transitions $t, \Psi_{\text {first }}(\sigma)(t)=1$ if $\bullet^{\bullet} \neq \bullet^{\bullet} t$ for all transitions $\bar{t}$ in front of the first occurrence of $t$ in $\sigma$, and $\Psi_{\text {first }}(\sigma)(t)=0$ otherwise. For $\sigma, \tau \in T^{*}, \sigma \doteq \tau \in T^{*}$ is obtained by deleting the first $\min \{\Psi(\sigma)(t), \Psi(\tau)(t)\}$ occurences of each transition $t$ from $\sigma$.

If there is a marking $\mu^{\prime}$ with $\mu \stackrel{\sigma}{\rightarrow} \mu^{\prime}$, then we say that $\sigma$ (the Parikh vector $\Psi(\sigma)$, resp.) is enabled at $\mu$ and leads from $\mu$ to $\mu^{\prime}$. For a marked Petri net $\left(N, \mu^{(0)}\right)$, we call a transition sequence that is enabled at $\mu^{(0)}$ a firing sequence. A marking $\mu$ is called reachable if $\mu^{(0)} \stackrel{\sigma}{\rightarrow} \mu$ for some $\sigma$. The reachability set $\mathcal{R}\left(N, \mu^{(0)}\right)$ of $\left(N, \mu^{(0)}\right)$ consists of all reachable markings. We say that a marking $\mu$ can be covered if there is a reachable marking $\mu^{\prime} \geq \mu$.

The displacement $\Delta: \mathbb{N}_{0}^{m} \rightarrow \mathbb{Z}^{n}$ maps Parikh vectors $\Phi \in \mathbb{N}_{0}^{m}$ onto the change of tokens at the places $p_{1}, \ldots, p_{n}$ when applying transition sequences with Parikh image $\Phi$. That is, we have $\Delta(\Phi)(p)=\sum_{t \in T} \Phi(t) \cdot(F(t, p)-F(p, t))$ for all places $p$. Accordingly, we define the displacement $\Delta(\sigma)$ of a transition sequence $\sigma$ by $\Delta(\sigma):=\Delta(\Psi(\sigma))$.

A Parikh vector or a transition sequence having nonnegative displacement at all places is called a nonnegative loop since, if it is fired at some marking, the loop can immediately be fired again at the resulting marking. A nonnegative loop having positive displacement at some place $p$ is a positive loop (for $p$ ). A nonnegative loop with displacement 0 at all places is a zero-loop. For a marking $\mu$, a transition sequence $\sigma$, and a subset $S \subseteq P$ of places, we define $\max (\mu, S):=$ $\max _{p \in S} \mu(p)$, and $\max (\mu):=\max (\mu, P)$, as well as $\max (\mu, \sigma, S):=\max _{i \in[0,|\sigma|]} \max \left(\mu+\Delta\left(\sigma_{(i)}\right), S\right)$, and $\max (\mu, \sigma):=\max (\mu, \sigma, P)$.

The wipe-extension $\mathcal{P}^{-}=\left(P, T^{-}, F^{-}\right)$of a Petri net $\mathcal{P}=(P, T, F)$ is obtained from $\mathcal{P}$ by introducing, for each place $p_{i} \in P$, a transition $t_{i}^{-}$with $F^{-}\left(p_{i}, t_{i}^{-}\right)=1$.

Some marked Petri nets have reachability sets that are semilinear. A set $S \subseteq \mathbb{N}_{0}^{n}$ is semilinear, if there are a $k \in \mathbb{N}_{0}$ and linear sets $L_{1}, \ldots, L_{k} \subseteq \mathbb{N}_{0}^{n}$ such that $S=\bigcup_{i \in[k]} L_{i}$. A set $L \subseteq \mathbb{N}_{0}^{n}$ is linear, if there are $\ell \in \mathbb{N}_{0}$ and vectors $b, p_{1}, \ldots, p_{\ell} \in \mathbb{N}_{0}^{n}$ such that $L=\left\{b+\sum_{i \in[\ell]} a_{i} p_{i} \mid a_{i} \in \mathbb{N}_{0}, i \in[\ell]\right\}$. The vector $b$ is the constant vector of $L$, while the vectors $p_{i}$ are the periods of $L$. A semilinear representation of a semilinear set $S$ is a set consisting of $k$ pairs $\left(b_{i},\left\{p_{i, 1}, \ldots, p_{i, \ell_{i}}\right\}\right), i \in[k]$, for some $k \in \mathbb{N}_{0}$, such that $S=\bigcup_{i \in[k]} L_{i}$ where $L_{i}=\left\{b_{i}+\sum_{j \in\left[\ell_{i}\right]} a_{i, j} p_{i, j} \mid a_{i, j} \in \mathbb{N}_{0}, j \in\left[\ell_{i}\right]\right\}$. If two Petri nets allow the construction of semilinear representations of the respective reachability sets within a certain space bound, then many problems are decidable that are undecidable for Petri nets in general, and space bounds can be given as well. We will use this well known approach for the containment and the equivalence problems.

Throughout this paper we use a succinct encoding scheme. Every number is encoded in binary representation. A Petri net is encoded as an enumeration of places $p_{1}, \ldots, p_{n}$ and transitions $t_{1} \ldots, t_{m}$ followed by an enumeration of the edges with their respective edge weight. A vector of $\mathbb{N}_{0}^{k}$ is encoded as a $k$-tuple. If we regard a tuple as an input (e.g. a marked Petri net), then it is encoded as a tuple of the encodings of the particular components. size $(\mathcal{P})$ denotes the encoding size of a marked Petri net $\mathcal{P}$. Analogously, $\operatorname{size}(\mathcal{P}, \mu)$ is the encoding size of $\mathcal{P}$ together with an additional marking $\mu$.

In this paper, we study the following problems for gcf-PNs. 
- RecLFS: Given a gcf-PN $\mathcal{P}$ and a Parikh vector $\Phi$, is $\Phi$ enabled in $\mathcal{P}$ ?

- Reachability: Given a gcf-PN $\mathcal{P}$ and a marking $\mu$, is $\mu$ reachable in $\mathcal{P}$ ?

- Zero-Reachability: Given a gcf-PN $\mathcal{P}$, is the empty marking reachable in $\mathcal{P}$ ?

- Covering: Given a gcf-PN $\mathcal{P}$ and a marking $\mu$, is $\mu$ coverable in $\mathcal{P}$ ?

- Boundedness: Given a gcf-PN $\mathcal{P}$, is there, for each $k \in \mathbb{N}$, a reachable marking $\mu$ with $\max (\mu) \geq k$ ?

- Containment: Given two gcf-PNs $\mathcal{P}$ and $\mathcal{P}^{\prime}$, is $\mathcal{R}(\mathcal{P}) \subseteq \mathcal{R}\left(\mathcal{P}^{\prime}\right)$ ?

- Equivalence: Given two gcf-PNs $\mathcal{P}$ and $\mathcal{P}^{\prime}$, is $\mathcal{R}(\mathcal{P})=\mathcal{R}\left(\mathcal{P}^{\prime}\right)$ ?

We remark that the input size of a problem instance consists of the encodings of all entities that are declared as being "given" in the respective problem statement.

\section{PSPACE-Hardness of the RecLFS, (Zero-)Reachability, Covering, and Boundedness Problems of gss-PNs}

In this section, we show PSPACE-hardness for the (zero-)reachability, the covering, and the boundedness problems by illustrating how to simulate PSPACE-Turing machines (deciding decision problems) by gss-PNs. We give, for each problem $L \in \mathrm{PSPACE}$, a logspace reduction from $L$ to the problems mentioned above. To this end, we first describe the initial step that is shared by each of these reductions. After that, we will extend this initial step appropriately.

Consider an arbitrary language $L \in$ PSPACE over the alphabet $\Sigma$. Then there exists a deterministic Turing machine $M=\left(Q, \Gamma, \square, \Sigma, \delta, q_{0}, q_{\text {acc }}\right)$ with state set $Q$, tape alphabet $\Gamma \supsetneqq \Sigma$, blank symbol $\square \in \Gamma \backslash \Sigma$, transition relation $\delta \subseteq Q \backslash\left\{q_{\text {acc }}\right\} \times \Gamma \times Q \times \Gamma \times\{-1,0,1\}$, initial state $q_{0} \in Q$ and accepting state $q_{\text {acc }} \in Q$ such that, w.l.o.g., on input $x \in \Sigma^{*}, M$ exhibits the following behavior:

- $M$ only uses the first $\left\lceil(|x|+2)^{c_{1}}\right\rceil$ tape positions at each step of the computation, for some constant $c_{1}$.

- At the beginning, the tape contains the word $x$ in the first $|x|$ positions. All other positions contain $\square$.

- $M$ halts after at most $\left\lceil 2^{(|x|+2)^{c_{2}}}\right\rceil$ steps, for some constant $c_{2}$.

- $M$ halts in state $q_{\text {acc }}$ with the first $\left\lceil(|x|+2)^{c_{1}}\right\rceil$ tape positions being $\square$ and the head over the first tape position if and only if $x \in L$.

We now describe the gss-PN $\mathcal{P}=\left(P, T, F, \mu^{(0)}\right)$, onto which the input word $x$ is mapped. For a better understanding, the reader is encouraged to simultaneously consult Figure 1 which illustrates (part of) this construction.

The set of places can be written as a union $P=\bigcup_{i \in\left[\left[(|x|+2)^{c_{1}}\right\rceil\right]} P_{i} \cup \tilde{P} \cup\left\{p_{\text {acc }}\right\}$ of disjoint sets of places. The set $P_{i}$ contains a place $p_{(s @ i)}^{(\mathrm{symb})}$ for each symbol $s \in \Gamma$ encoding the contents of the tape, and places $p_{(d @ i)}^{(\text {req })}$ and $p_{(d @ i)}^{(\text {exec })}$ for each transition $d \in \delta$, encoding the execution of $d$ with the head of $M$ at position $i$. The set $\tilde{P}$ contains a place $p_{(\operatorname{pos}=i)}^{(\text {state }=q)}$ for each state $q \in Q$ and tape position $i \in\left[\left\lceil(|x|+2)^{c_{1}}\right\rceil\right]$, encoding the current state of $M$ and the position of the head. The place $p_{\text {acc }}$ encodes whether $M$ accepts $x$. Next, we define the transitions. If not stated otherwise, the edge multiplicities are 1. For each tape position $i \in\left[\left\lceil(|x|+2)^{c_{1}}\right\rceil\right]$ and transition $d=\left(q, s, q^{\prime}, s^{\prime}, h\right) \in \delta$ of $M$, there are five transitions representing the execution of $d$ with the head at position $i$.

- $t_{(d @ i)}^{(\text {req state \& pos) }}$ from $p_{(\text {pos }=i)}^{(\text {state }=q)}$ to $p_{(d @ i)}^{(\text {req })}$. The outgoing edge has multiplicity 3. 


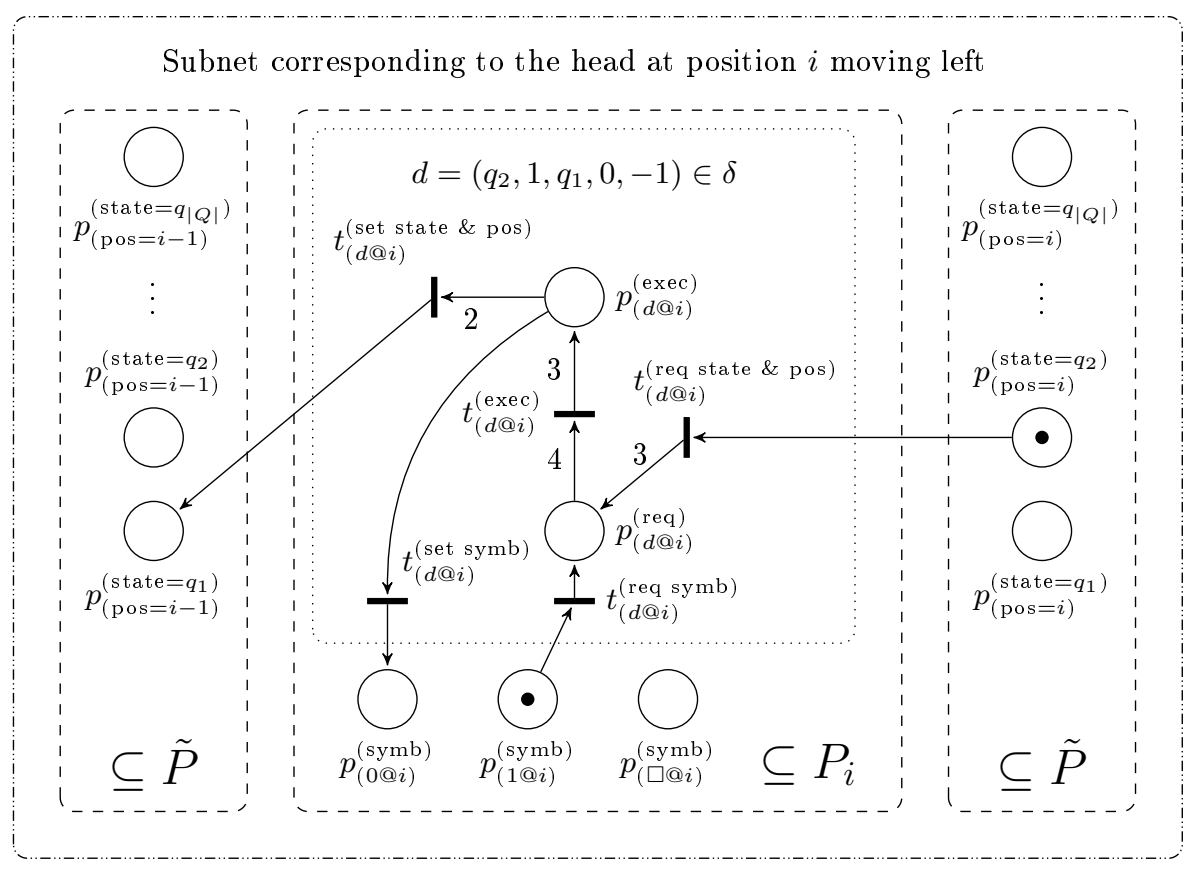

Figure 1: This figure illustrates a subnet of $\mathcal{P}$ where the marking is a configuration marking. The middle dashed rectangle contains (a subset of) the places and transitions corresponding to tape position $i$. The dotted rectangle contains the places and transitions corresponding to the transition $d=\left(q_{2}, 1, q_{1}, 0,-1\right) \in \delta$ of the Turing machine $M$. This transition requires that $M$ is in state $q_{2}$, and that the tape contains the symbol 1 at the position $i$ of the head. When $d$ is executed, $M$ switches into state $q_{1}$, writes 0 onto the tape, and moves to the left. This behavior is simulated by the transitions inside of the dotted rectangle (see Lemma 3.2 ).

- $t_{(d @ i)}^{(\mathrm{req} \mathrm{symb})}$ from $p_{(s @ i)}^{(\mathrm{symb})}$ to $p_{(d @ i)}^{(\mathrm{req})}$.

- $t_{(d @ i)}^{(\mathrm{exec})}$ from $p_{(d @ i)}^{(\mathrm{req})}$ to $p_{(d @ i)}^{(\mathrm{exec})}$. The multiplicities are 4 and 3.

- $t_{(d @ i)}^{(\text {set state \& pos) }}$ from $p_{(d @ i)}^{(\text {exec })}$ to $p_{\left(\text {pos }=i^{\prime}\right)}^{\left(\text {state }=q^{\prime}\right)}$. The incoming edge has multiplicity 2.

- $t_{(d @ i)}^{(\mathrm{set} \mathrm{symb})}$ from $p_{(d @ i)}^{(\mathrm{exec})}$ to $p_{\left(s^{\prime} @ i\right)}^{(\mathrm{symb})}$.

For recognizing acceptance of $x$ by $M$, there are the following transitions.

- There is a transition $t_{(\text {acc state })}$ from $p_{(\operatorname{pos}=1)}^{\left(\mathrm{state}=q_{\text {acc }}\right)}$ to $p_{\text {acc }}$. The outgoing edge has multiplicity 3 .

- For each tape position $i \in\left[\left\lceil(|x|+2)^{c_{1}}\right\rceil\right]$, there is a transition $t_{(\mathrm{acc} \mid i)}$ from $p_{(\square @ i)}^{(\mathrm{symb})}$ to $p_{\text {acc }}$.

Note that $\mathcal{P}$ is indeed a gss-PN. The initial marking $\mu^{(0)}$ of $\mathcal{P}$ has a token at $p_{(\text {pos }=1)}^{\left(\text {state }=q_{0}\right)}$, a token at $p_{\left(x_{i} @ i\right)}^{(\mathrm{symb})}$ for all $i \in[|x|]$, and a token at $p_{(\square @ i)}^{(\mathrm{symb})}$ for all $i>|x|$. All remaining places are empty.

We call a marking $\mu$ of $\mathcal{P}$ a configuration marking if it corresponds to a configuration of $M$, i.e., if the following holds.

- For each $i \in\left[\left\lceil(|x|+2)^{c_{1}}\right\rceil\right]$, there is exactly one $s \in \Gamma$ s.t. $\mu\left(p_{(s @ i)}^{(\mathrm{symb})}\right)=1$.

- There is exactly one $i \in\left[\left\lceil(|x|+2)^{c_{1}}\right\rceil\right]$ and exactly one $q \in Q$ such that $\mu\left(p_{(\operatorname{pos}=i)}^{(\text {state }=q)}\right)=1$. 
- All other places are empty.

Note that, for a configuration marking, the places $p_{(s @ i)}^{(\mathrm{symb})}$ and $p_{(\mathrm{pos}=i)}^{(\mathrm{state}=q)}$ contain either 0 or 1 tokens, i.e., they can be considered binary variables. In the obvious way, these places encode the contents of the tape as well as the state and the position of the head of $M$. For a configuration marking $\mu$ of $\mathcal{P}, \operatorname{conf}(\mu)$ denotes the corresponding configuration of $M$.

The marking $\mu^{\text {acc }}$ is the configuration marking of the unique accepting configuration of $M$, i.e., $\mu^{\text {acc }}\left(p_{(\text {pos }=1)}^{\left(\text {state }=q_{\text {acc }}\right)}\right)=1, \mu^{\text {acc }}\left(p_{(\square @ i)}^{(\text {symb })}\right)=1$ for all $i \in\left[\left\lceil(|x|+2)^{c_{1}}\right\rceil\right]$, and all other places are empty. Note that $\operatorname{conf}\left(\mu^{(0)}\right)$ is the initial configuration of $M$. Moreover, $\mu^{\text {end }}$ is the marking that has $\left\lceil(|x|+2)^{c_{1}}\right\rceil+3$ tokens at $p_{\text {acc }}$, and all other places are empty.

For a simpler description of reachable markings, we characterize them by certain types.

(1) Type A: Each $P_{i}, i \in\left[\left\lceil(|x|+2)^{c_{1}}\right\rceil\right]$, contains exactly one token, $\tilde{P}$ contains exactly one token, and $p_{\text {acc }}$ is empty.

(2) Type B: There is a $j \in\left[\left\lceil(|x|+2)^{c_{1}}\right\rceil\right]$ such that each $P_{i}, i \in\left[\left\lceil(|x|+2)^{c_{1}}\right\rceil\right] \backslash\{j\}$, contains exactly one token, and $\tilde{P}$ and $p_{\text {acc }}$ are empty. Furthermore, the possible markings of $P_{j}$ are exactly those which can be obtained by starting with 3 tokens at exactly one of the places $p_{(d @ i)}^{(\text {req) }}, d \in \delta$, and with one additional token at exactly one place of $P_{j}$ (therefore, 4 tokens at some place $p_{(d @ i)}^{(\mathrm{req})}$ is a possibility), and then firing transitions which transfer tokens within $P_{j}$.

Lemma 3.1. Let $\mu^{(0)}$ be a configuration marking. Then, each marking $\mu$ reached by a firing sequence $\rho$ such that $t_{(\text {acc state) }}, t_{(\mathrm{acc} \mid i)} \notin \rho$ for all $i \in\left[\left\lceil(|x|+2)^{c_{1}}\right\rceil\right]$ is either of type $A$ or type $B$.

Proof. This lemma can be proven by induction on the length of $\rho: \mu^{(0)}$ is a marking of type A. Let $\rho$ have length $k$ and assume the validity of the claim for all such firing sequences of length less than $k$. Let $\mu^{\prime}$ be the marking reached by $\rho_{(1,|\rho|-1)}$. If $\mu^{\prime}$ is of type $\mathrm{A}$, then firing $t_{(d @ i)}^{(\text {req state \& pos })}$ for the single possible $i \in\left[\left\lceil(|x|+2)^{c_{1}}\right\rceil\right]$ yields a marking of type B. Firing any other transition (except $t_{(\text {acc state) }}$ and $\left.t_{(\mathrm{acc} \mid i)}\right)$ yields a marking of type $\mathrm{A}$ again. If $\mu^{\prime}$ is of type $\mathrm{B}$, then firing $t_{(d @ i)}^{\text {(set state \& pos) }}$ for the single possible $i \in\left[\left\lceil(|x|+2)^{c_{1}}\right\rceil\right]$ yields a marking of type A. Firing any other transition (except $t_{(\text {acc state) }}$ and $t_{(\text {acc } \mid i)}$ ) yields a marking of type $\mathrm{B}$ again.

Lemma 3.2. Let $\mu$ be a configuration marking of $\mathcal{P}$. Then the transition quintuple

$$
t_{(d @ i)}^{(\text {req state \& pos })} \cdot t_{(d @ i)}^{(\text {req symb })} \cdot t_{(d @ i)}^{(\text {exec })} \cdot t_{(d @ i)}^{(\text {set state \& pos })} \cdot t_{(d @ i)}^{(\text {set symb })}
$$

is enabled at $\mu$ in $\mathcal{P}$ if and only if the transition $d \in \delta$ is enabled at conf $(\mu)$ in $M$. If this quintuple is enabled, then, for marking $\mu^{\prime}$ reached by this quintuple, conf $\left(\mu^{\prime}\right)$ is the configuration of $M$ obtained by applying d at conf $(\mu)$.

Proof. Clear.

We now show how firing sequences of $\mathcal{P}$ leading to configuration markings and computation paths of $M$ correspond to each other.

Lemma 3.3. Let $\mu, \mu^{\prime}$ be configuration markings of $\mathcal{P}$. Then, $\mu \stackrel{\sigma}{\rightarrow} \mu^{\prime}$ in $\mathcal{P}$ for some transition sequence $\sigma$ if and only if conf $\left(\mu^{\prime}\right)$ is reachable from $\operatorname{conf}(\mu)$ in $M$.

Proof. " $\Leftarrow$ ": This immediately follows from Lemma 3.2

" $\Rightarrow$ ": In the following, we will often make use of Lemma 4.2 without explicitly referring to it. For $\mu=\mu^{\prime}$, the claim holds. Let $k>0$, and assume that the claim holds for all configuration markings $\mu$ and $\mu^{\prime}$ such that the shortest $\sigma$ with $\mu \stackrel{\sigma}{\rightarrow} \mu^{\prime}$ has $|\sigma|<k$. Now, consider two configuration markings $\mu, \mu^{\prime}$ such that there is a transition sequence $\sigma$ with $\mu \stackrel{\sigma}{\rightarrow} \mu^{\prime}$ and $|\sigma|=k$. First, note that $t_{(\text {acc state) }}, t_{(\text {acc } \mid i)} \notin \sigma$ for all $i \in\left[\left\lceil(|x|+2)^{c_{1}}\right\rceil\right]$, since otherwise, $\mu^{\prime}$ wouldn't be a configuration marking. 
Let $i$ and $q$ be the unique index and state with $\mu\left(p_{(\operatorname{pos}=i)}^{(\text {state }=q)}\right)=1$. If $t_{(d @ i)}^{(\text {req state \& pos })} \notin \Psi_{\text {first }}(\sigma)$, then $\mu^{\prime}$ cannot be a configuration marking since there is a place $p_{\left(d @ i^{\prime}\right)}^{(\mathrm{req})}$ where tokens get stuck. Hence, $t_{(d @ i)}^{\text {(req state \& pos })} \in \Psi_{\text {first }}(\sigma)$, and we can push this transition to the front of the firing sequence, yielding a permutation $\rho$ of $\sigma$ with $\rho_{(1)}=t_{(d @ i)}^{(\text {req state \& pos })}$ and $\rho_{(2, k)}=\sigma \cdot t_{(d @ i)}^{(\text {req state \& pos })}$ that is enabled at $\mu$. With the same argument, we can push $t_{(d @ i)}^{(\text {req symb })}$ and $t_{(d @ i)}^{(\text {exec })}$ to the position right behind $t_{(d @ i)}^{(\text {req state \& pos })}$ yielding a permutation $\varphi$ of $\rho$ with $\varphi_{(1,3)}=t_{(d @ i)}^{\text {(req state } \& \text { pos })} \cdot t_{(d @ i)}^{(\text {req symb })}$. $t_{(d @ i)}^{(\text {exec })}$ and $\varphi_{(4, k)}=\rho \cdot t_{(d @ i)}^{(\text {req symb })} \cdot t_{(d @ i)}^{(\text {exec })}$ that is enabled at $\mu$.

Assume for the sake of contradiction that the first two transitions $t, t^{\prime}$ of $\varphi_{(4, k)}$ with $\bullet=t^{\bullet} t^{\prime}=$ $p_{(d @ i)}^{(\text {exec })}$, which must exist, are both $t=t^{\prime}=t_{(d @ i)}^{(\text {set symb })}$ or $t=t^{\prime}=t_{(d @ i)}^{(\text {set state \& pos })}$. In the first case, we can move them both to the positions right behind $\varphi_{(1,3)}$ yielding a permutation $\psi$ of $\varphi$. But now, after firing $\psi_{(5)}$, no place $p_{\left(d^{\prime} @ i^{\prime}\right)}^{(\mathrm{req})}$ can ever gain at least 4 tokens anymore, preventing the firing of any transition $t_{\left(d^{\prime} @ i^{\prime}\right)}^{(\mathrm{exec})}$ and subsequently $t_{\left(d^{\prime} @ i^{\prime}\right)}^{(\text {set state \& pos) }}$. Hence, $\psi$ cannot lead to a configuration marking. This contradiction shows that this case cannot occur. In the second case, we can move $t_{(d @ i)}^{(\text {set state \& pos) }}$ to the position right behind $\varphi_{(1,3)}$ yielding a permutation $\psi$ of $\varphi$. Now, $\psi_{(5, k)}$ must have a prefix that contains $t_{(d @ i)}^{(\text {exec })}$ but neither $t_{(d @ i)}^{\text {(set symb) }}$ nor $t_{(d @ i)}^{(\text {set state \& pos })}$. By firing this transition $t_{(d @ i)}^{(\mathrm{exec})}$, we obtain a marking where $p_{(d @ i)}^{(\mathrm{exec})}$ has (at least) 4 tokens. From this marking, we can fire $t_{(d @ i)}^{\text {(set state \& pos) }}$ two times in a row, yielding a marking that is neither of type A nor of type B, a contradiction to Lemma 3.1. Hence, the second case is also impossible.

We conclude that $t$ and $t^{\prime}$ are different. Moving them to the fourth and fifth position yields a permutation $\psi$ of $\varphi$ that is enabled at $\mu$ whose first five transitions are a transition quintuple. Let $\mu \stackrel{\psi_{(5)}}{\longrightarrow} \mu^{\prime \prime} \stackrel{\psi_{(6, k)}}{\longrightarrow} \mu^{\prime}$. By Lemma $3.2, \mu^{\prime \prime}$ is a configuration marking such that transition $d \in \delta$ of $M$ leads from $\operatorname{conf}(\mu)$ to $\operatorname{conf}\left(\mu^{\prime \prime}\right)$. Furthermore, by the induction assumption, $M$ has a computation path leading from $\operatorname{conf}\left(\mu^{\prime \prime}\right)$ to $\operatorname{conf}\left(\mu^{\prime}\right)$. This concludes the proof.

Lemma 3.4. The only reachable marking $\mu$ with $\mu\left(p_{\text {acc }}\right) \geq\left\lceil(|x|+2)^{c_{1}}\right\rceil+3$ is $\mu^{\text {end }}$. Moreover, $\mu^{\text {end }}$ is reachable if and only if $\mu^{a c c}$ is reachable.

Proof. Let $\sigma$ be a firing sequence leading to a marking $\mu$ with $\mu\left(p_{\text {acc }}\right) \geq\left\lceil(|x|+2)^{c_{1}}\right\rceil+3$. Then, by pushing all occurrences of the transitions $t_{(\text {acc state) }}$ and $t_{(\text {acc } \mid i)}, i \in\left[\left\lceil(|x|+2)^{c_{1}}\right\rceil\right]$, to the end of the sequence which then constitute a suffix $\psi$ we obtain a firing sequence $\rho \cdot \psi$ that is a permutation of $\sigma$. Let $\mu^{\rho}$ be the marking reached by $\rho$. By Lemma 3.1 $\mu^{\rho}$ is of type A or B. The only such marking that has enough tokens to reach $\mu$ is $\mu^{\text {acc }}$, hence $\mu^{\rho}=\mu^{\text {acc }}$ is reachable. It immediately follows that $\mu=\mu^{\text {end }}$.

Lemma 3.5. The RecLFS, the zero-reachability, the reachability, the covering, and the boundedness problems of gss-PNs are PSPACE-hard.

Proof. We obtain the gss-PN $\mathcal{P}_{1}$ from $\mathcal{P}$ by adding transition $t$ with $F\left(p_{\text {acc }}, t\right)=\left\lceil(|x|+2)^{c_{1}}\right\rceil+3$. By Lemmata 3.3, 3.4, and 3.1, the following are equivalent.

- $M$ accepts $x$.

- $\mu^{\text {acc }}$ is reachable in $\mathcal{P}$.

- $\mu^{\text {end }}$ is coverable in $\mathcal{P}$.

- $\mu^{\text {end }}$ is reachable in $\mathcal{P}$.

- the empty marking is reachable in $\mathcal{P}_{1}$. 
This shows the PSPACE-hardness of the (zero-)reachability, and the covering problems.

We obtain $\mathcal{P}_{2}$ from $\mathcal{P}$ by adding a transition $t$ with $F\left(p_{\text {acc }}, t\right)=\left\lceil(|x|+2)^{c_{1}}\right\rceil+3$ and $F\left(t, p_{\text {acc }}\right)=$ $\left(\left\lceil(|x|+2)^{c_{1}}\right\rceil+3\right)^{2}$. By Lemmata 3.4 and 3.1 all places of $\mathcal{P}_{2}$ other than $p_{\text {acc }}$ are bounded, and $p_{\text {acc }}$ can obtain at least $\left\lceil(|x|+2)^{c_{1}}\right\rceil+3$ tokens if and only if $M$ accepts $x$. Therefore, $\mathcal{P}_{2}$ is unbounded if and only if $M$ accepts $x$. This proves PSPACE-hardness for the unboundedness and thereby for the boundedness problem.

We obtain $\mathcal{P}_{3}$ from $\mathcal{P}_{2}$ by adding, for each place $p$ of $\mathcal{P}_{2}$, a transition $t$ with $F\left(p_{\text {acc }}, t\right)=$ $\mu^{\text {end }}\left(p_{\text {acc }}\right)=\left\lceil(|x|+2)^{c_{1}}\right\rceil+3$ and $F(t, p)=\left(\left\lceil(|x|+2)^{c_{1}}\right\rceil+3\right) \cdot 4\left\lceil 2^{(|x|+2)^{c_{2}}}\right\rceil$. Then, by Lemma 3.4 the fact that $M$ is deterministic, and the bound on the running time of $M, M$ accepts $x$ if and only if the Parikh vector having $\left\lceil 2^{(|x|+2)^{c_{2}}}\right\rceil$ at each component is enabled in $\mathcal{P}_{3}$. This proves PSPACE-hardness for the RecLFS problem.

\section{Canonical Permutations, and the RecLFS, (Zero-)Reach- ability, and Covering Problems}

In this section, we first show PSPACE-completeness of the RecLFS problem. Then, we describe a procedure that, given a gcf-PN $\mathcal{P}=\left(P, T, F, \mu^{(0)}\right)$, and a firing sequence $\sigma$ with $\mu^{(0)} \stackrel{\sigma}{\rightarrow} \mu$, produces a permutation $\sigma^{\prime}$ of $\sigma$ enabled at $\mu^{(0)}$ such that every marking reached while firing $\sigma^{\prime}$ has encoding size polynomial in $\operatorname{size}(\mathcal{P}, \mu)$. We use these sequences to decide the reachability, and the covering problems in polynomial space, proving their PSPACE-completeness.

Theorem 4.1. The RecLFS problem of general Petri nets is PSPACE-complete, even if restricted to gss-PNs.

Proof. The PSPACE-hardness of the RecLFS problem of gss-PNs has been shown in Lemma 3.5. It remains to be shown that the RecLFS problem of general Petri nets is in PSPACE. Let $\mathcal{P}=\left(P, T, F, \mu^{(0)}\right)$ with largest edge multiplicity $W$ be a Petri net, and $\Phi$ a Parikh vector. Assume $\Phi$ is enabled, and let $\sigma$ be a firing sequence with $\Psi(\sigma)=\Phi$. Then, we have $\max \left(\mu^{(0)}, \sigma\right) \leq$ $\max \left(\mu^{(0)}\right)+|\sigma| W$, i.e., to encode the markings obtained while firing $\sigma$, we only need a polynomial number of bits. Therefore, the reachability problem of gcf-PNs is accepted in polynomial space by some Turing machine guessing $\sigma$ step by step. This proves that the reachability problem of gcf-PNs is in NPSPACE which, by Savitch's theorem [20], equals PSPACE.

Next, we propose four essential lemmata for the construction of canonical permutations of firing sequences in gcf-PNs.

Lemma 4.2. Let $\sigma$ be a firing sequence of a gcf-PN $\left(N, \mu^{(0)}\right)$. If $t \in \Psi_{\text {first }}\left(\sigma_{(i+1,|\sigma|)}\right)$ is enabled at $\mu^{(0)}+\Delta\left(\sigma_{(i)}\right)$, then $\sigma_{(i)} \cdot t \cdot\left(\sigma_{(i+1,|\sigma|)}-t\right)$ is a firing sequence.

Proof. Assume $\bullet \neq \emptyset$. Let $\sigma_{j}, j \geq i+1$, be the first occurrence of $t$ in $\sigma_{(i+1,|\sigma|)}$, and assume that $t$ is enabled at $\mu^{(0)}+\Delta\left(\sigma_{(i)}\right)$. Then, $t$ is enabled at $\mu^{(0)}+\Delta\left(\sigma_{(j-1)}\right)$ since, by the choice of $t$, $\Delta\left(\sigma_{(j-1)}\right)(\bullet t) \geq \Delta\left(\sigma_{(i)}\right)(\bullet t)$. Furthermore, since $\bullet^{\bullet} \sigma_{j-1} \neq \bullet^{\bullet} t, \sigma_{j-1}$ is enabled at $\mu+\Delta\left(\sigma_{(j-2)} \cdot t\right)$. If $\bullet t=\emptyset$, a similar argumentation can be applied. By iteratively performing pairwise switches, we obtain the Lemma.

Lemma 4.3. Let $(P, T, F)$ be a gcf-PN, $\sigma$ a transition sequence, and $\mu, \mu^{\prime}$ markings with $\mu+$ $\Delta(\sigma)=\mu^{\prime}$ and $\mu(p), \mu^{\prime}(p) \geq W$ for all $p \in \bullet^{\bullet} \sigma$. Then, there is a permutation of $\sigma$ enabled at $\mu$ (and leading to $\mu^{\prime}$ ).

Proof. For the empty sequence $\sigma$ and all markings $\mu=\mu^{\prime}$, the claim holds. Let $\sigma$ be a transition sequence of length $k>0$ and $\mu, \mu^{\prime}$ be markings satisfying the requirements.

Assume, the claim holds for all transition sequences of length less than $k$ and for all markings satisfying the requirements. We initialize $\tilde{\sigma} \leftarrow()$ and $\bar{\sigma} \leftarrow \sigma$. As long as $|\tilde{\sigma}|<k$ and there is a place $p \in \bullet^{\bullet} \bar{\sigma}$ with $\Delta(\tilde{\sigma})(p) \geq 0$, we choose a transition $t \in \bar{\sigma}$ with ${ }^{\bullet} t=p$, and set $\tilde{\sigma} \leftarrow \tilde{\sigma} \cdot t$ as well as $\bar{\sigma} \leftarrow \bar{\sigma} \bullet t$. 
At the end of this procedure, $\tilde{\sigma}$ is a nonempty transition sequence enabled at $\mu$. If $\tilde{\sigma}$ has length $k$, then we are finished.

Otherwise, we have $0<|\tilde{\sigma}|<k$. Then, $\tilde{\sigma}$ satisfies $\Delta(\tilde{\sigma})(p) \in[-W,-1]$ for all $p \in \bullet^{\bullet} \bar{\sigma}$. Consider the sequence $\bar{\sigma}$, and let $\mu^{\bar{\sigma}}=\mu+\Delta(\bar{\sigma})$. Since $|\bar{\sigma}|<k$, and $\mu^{\bar{\sigma}}(p)=(\mu+\Delta(\tilde{\sigma} \cdot \bar{\sigma})-\Delta(\tilde{\sigma}))(p)=$ $\left(\mu^{\prime}-\Delta(\tilde{\sigma})\right)(p)>\mu^{\prime}(p) \geq W$ for all $p \in \bullet \bar{\sigma}$, we can apply the induction hypothesis to $\bar{\sigma}, \mu$ and $\mu^{\bar{\sigma}}$. Hence, $\mu \stackrel{\bar{\sigma}^{\prime}}{\rightarrow} \mu^{\bar{\sigma}}$ for some permutation $\bar{\sigma}^{\prime}$ of $\bar{\sigma}$.

In addition to $\mu^{\bar{\sigma}}(p) \geq W$ for all $p \in \bullet^{\bar{\sigma}}$, we have $\mu^{\bar{\sigma}}(p)=\mu(p) \geq W$ for all $p \in \bullet^{\bullet} \backslash \backslash \bullet \bar{\sigma}$, and thus $\mu^{\bar{\sigma}}(p) \geq W$ for all $p \in \bullet \tilde{\sigma}$. By applying the induction hypothesis to $\tilde{\sigma}, \mu^{\bar{\sigma}}$ and $\mu^{\prime}$, we obtain $\mu^{\bar{\sigma}} \stackrel{\tilde{\sigma}^{\prime}}{\rightarrow} \mu^{\prime}$ for some permutation $\tilde{\sigma}^{\prime}$ of $\tilde{\sigma}$. Therefore, the permutation $\bar{\sigma}^{\prime} \cdot \tilde{\sigma}^{\prime}$ of $\sigma$ is enabled at $\mu$ and leads from $\mu$ to $\mu^{\prime}$.

Lemma 4.4. Let $\mathcal{P}=(P, T, F)$ be a gcf-PN with largest edge multiplicity $W$, and $S \subseteq P$ a subset of places. Further, let $\sigma=\sigma_{1} \cdots \sigma_{k}, \sigma_{i} \in T$, be a transition sequence of $\mathcal{P}$ with $\mu^{(0)} \stackrel{\sigma_{1}}{\longrightarrow}$ $\mu^{(1)} \ldots \mu^{(k-1)} \stackrel{\sigma_{k}}{\longrightarrow} \mu^{(k)}$ such that

$(a) \cdot \sigma \subseteq S$,

(b) $\mu^{(i-1)}\left(\bullet_{i}\right)=\max \left(\mu^{(i-1)}, S\right)$ for all $i \in[k]$ (i.e., each transition removes tokens from a place of $S$ with the maximum number of tokens), and

(c) $\max \left(\mu^{(k)}, S\right)>\max \left(\mu^{(0)}, S\right)+2|S| W$.

Then, for some $i \in[1, k-1]$, the suffix $\sigma_{(i, k)}$ is a positive loop.

Proof. Consider the disjoint intervals $\left[\max \left(\mu^{(0)}, S\right)+2 \ell W-2 W+1, \max \left(\mu^{(0)}, S\right)+2 \ell W\right], \ell \in[|S|]$. Since $\max \left(\mu^{(k)}, S\right)$ is outside of all of these intervals, at least one of these intervals, denoted by $[a, b]$, must satisfy $\mu^{(k)}(p) \notin[a, b]$ for all $p \in S$. Let $i \in[0, k-1]$ be the smallest index such that $\max \left(\mu^{(j)}, S\right) \geq a+W$ for all $j \in[i, k]$. (Note that this index exists since $\max \left(\mu^{(k-1)}, S\right) \geq$ $\max \left(\mu^{(k)}, S\right)-W \geq b-W+1=a+W$.) We observe $\max \left(\mu^{(i)}, S\right) \leq b$ since, by the choice of $i, \max \left(\mu^{(i-1)}, S\right) \leq a+W-1=b-W$. Now, for all $p \in S$ having a $j \in[i, k-1]$ with $\mu^{(j)}(p) \in[a, b]$, we observe $\mu^{(k)}(p)>b$, and therefore $\mu^{(i)}(p)<\mu^{(k)}(p)$, since, by the choice of $i$, the token numbers of these places can leave the interval $[a, b]$ only by crossing the border $b$. (This applies to at least one place of $S$.) For all other places, we have $\mu^{(i)}(p) \leq \mu^{(k)}(p)$ since the remaining sequence leading from $\mu^{(i)}$ to $\mu^{(k)}$ doesn't remove tokens from them. Therefore, $\sigma_{(i+1, k)}$ is a positive loop.

Lemma 4.5. Let $N=(P, T, F)$ be a Petri net with $n$ places and $m$ transitions, and let $W$ be the largest edge multiplicity of $N$. Then, there is a finite set $\mathcal{H}(N)=\left\{\Phi^{(1)}, \ldots, \Phi^{(k)}\right\} \varsubsetneqq \mathbb{N}_{0}^{m}$ of nonnegative loops of $N$ such that each loop of $\mathcal{H}(N)$ consists of at most $(1+(n+m) W)^{n+m}$ transitions, and such that, for each nonnegative loop $\Phi$ of $N$, there are $a_{1}, \ldots, a_{k} \in \mathbb{N}_{0}$ with $\Phi=a_{1} \Phi^{(1)}+\ldots+a_{k} \Phi^{(k)}$.

Proof. Let $D \in \mathbb{Z}^{n \times m}$ be the displacement matrix of $\mathcal{P}$, i.e., the $i$-th column of $D$ equals $\Delta\left(t_{i}\right)$. Consider the system $D \Phi \geq \mathbf{0}$ of linear diophantine inequalities. The set $L \varsubsetneqq \mathbb{N}_{0}^{m}$ of nontrivial nonnegative integral solutions of this system equals the set of nonnegative loops having at least one transition. Now, consider the system $\left(D,-I_{n}\right) y=0$ having the set $L^{\prime} \varsubsetneqq \mathbb{N}_{0}^{m+n}$ of nontrivial solutions where $I_{n}$ is the $n \times n$-identity matrix.

By Theorem 1 of [19], this system has a set $\mathcal{H}\left(D,-I_{n}\right)$ of minimal solutions (called the Hilbert basis) having the following properties:

(i) Each nontrivial solution is a linear combination of the elements of $\mathcal{H}\left(D,-I_{n}\right)$ with nonnegative integral coefficients.

(ii) Each element of $\mathcal{H}\left(D,-I_{n}\right)$ has a component sum of at most $(1+(m+n) W)^{m+n}$. 
Let $y \in L^{\prime}$, and $a$ and $b$ be the projection of $y$ onto the first $m$ and the last $n$ components, respectively. We observe that $a$ is a nonnegative loop of $N$ with $\Delta(a)=b$. Moreover, the set of projections of the elements of $L^{\prime}$ onto the first $m$ components equals $L$. The properties (i) and (ii) for $L^{\prime}$ imply equivalent properties for $L$. This concludes the proof.

Using these lemmata, we can show that firing sequences have canonical permutations with nice properties.

Lemma 4.6. There is a constant $c$ such that, for each gcf-PN $\mathcal{P}=\left(P, T, F, \mu^{(0)}\right)$ and each firing sequence $\sigma$ leading from $\mu^{(0)}$ to $\mu$, there is a permutation $\varphi$ of $\sigma$ leading from $\mu^{(0)}$ to $\mu$, and satisfying $\max \left(\mu^{(0)}, \varphi\right) \leq\left(2 n m W+\max \left(\mu^{(0)}\right)+\max (\mu)\right)^{c(n+m)}$.

Proof. Let $\mathcal{P}=\left(P, T, F, \mu^{(0)}\right)$ be a gcf-PN, and $\sigma$ a firing sequence leading to some marking $\mu^{\sigma}$. We define two special levels $\ell_{\text {big }}:=\max \left\{W, \max \left(\mu^{(0)}\right), \max \left(\mu^{\sigma}\right)+1\right\}$ and $\ell_{\text {fire }}:=\ell_{\text {big }}+W$. Additionally, for $i \in[0, n]$, we define the levels $\ell_{i}:=\ell_{\text {fire }}+W+i \cdot\left(\max \left\{(1+(n+m) W)^{n+m}, 2 n\right\}+1\right) W$. A place $p$ is big at a marking $\mu$ if $\mu(p) \geq \ell_{\text {big }}$, and firing if $\mu(p) \geq \ell_{\text {fire }}$.

Consider the following invariants for two transition sequences $\tilde{\sigma}$ and $\bar{\sigma}$ :

(i) $\tilde{\sigma} \cdot \bar{\sigma}$ is a permutation of $\sigma$ with $\mu^{(0)} \stackrel{\tilde{\sigma}}{\rightarrow} \mu^{\tilde{\sigma}} \stackrel{\bar{\sigma}}{\rightarrow} \mu^{\sigma}$,

(ii) $\max \left(\mu^{(0)}, \tilde{\sigma}\right) \leq \ell_{n}$, and

(iii) if there are $b \geq 1$ big places at $\mu^{\tilde{\sigma}}$, then $\max \left(\mu^{\tilde{\sigma}}\right) \leq \ell_{b-1}$.

For $\tilde{\sigma}=()$ and $\bar{\sigma}=\sigma$, these invariants are obviously satisfied. Assume $|\tilde{\sigma}|<|\sigma|$, and that $\tilde{\sigma}$ and $\bar{\sigma}$ satisfy the invariants. We show how to extend $\tilde{\sigma}$ at the end to a longer transition sequence $\tilde{\sigma}^{\text {new }}$ and obtain a corresponding sequence $\bar{\sigma}^{\text {new }}$ such that $\tilde{\sigma}^{\text {new }}$ and $\bar{\sigma}^{\text {new }}$ again satisfy the invariants.

First, consider the case that there are no firing places at $\mu^{\tilde{\sigma}}$. Then, we set $\tilde{\sigma}^{\text {new }}:=\tilde{\sigma} \cdot \bar{\sigma}_{(1)}$, and $\bar{\sigma}^{\text {new }}:=\bar{\sigma}_{(2,|\bar{\sigma}|)}$. $\tilde{\sigma}^{\text {new }}$ and $\bar{\sigma}^{\text {new }}$ obviously satisfy property (i). For (ii) and (iii) notice that, for each big place $p$ of $\mu^{\tilde{\sigma}}+\Delta\left(\bar{\sigma}_{(1)}\right)$, we have $\left(\mu^{\tilde{\sigma}}+\Delta\left(\bar{\sigma}_{(1)}\right)\right)(p) \leq \mu^{\tilde{\sigma}}(p)+W<\ell_{\text {fire }}+W=\ell_{0}$.

Next, consider the case that there are firing places at $\mu^{\tilde{\sigma}}$. Let $S$ be the set of big places at $\mu^{\tilde{\sigma}}$ and $b=|S| \geq 1$ their number. The number of tokens of a big place $p^{*} \in S$ as a function of time is illustrated in (a) of Figure 2 . We initialize an empty transition sequence $\alpha \leftarrow$ (), as well as $\bar{\sigma}^{\prime} \leftarrow \bar{\sigma}$. As long as there is a firing place $p \in S$ at $\mu^{\tilde{\sigma}}+\Delta(\alpha)$, we select the transition $t \in \Psi_{\text {first }}\left(\bar{\sigma}^{\prime}\right)$ with $p=\bullet t$, and set $\alpha \leftarrow \alpha \cdot t$, as well as $\bar{\sigma}^{\prime} \leftarrow \bar{\sigma}^{\prime} \bullet t$. Notice that $t$ must exist since $\bar{\sigma}^{\prime}$ must reduce the number of tokens at $p$ in order to reach $\mu^{\sigma}(p)$. By Lemma $4.2 \tilde{\sigma} \cdot \alpha \cdot \bar{\sigma}^{\prime}$ is a firing sequence with $\mu^{(0)} \stackrel{\tilde{\sigma}}{\rightarrow} \mu^{\tilde{\sigma}} \stackrel{\alpha}{\rightarrow} \mu^{\alpha} \stackrel{\bar{\sigma}^{\prime}}{\rightarrow} \mu^{\sigma}$, and $\alpha$ is nonempty since $\mu^{\tilde{\sigma}}$ has a firing place, see (b) of Figure 2

Now, consider the nonnegative loop $\Phi$ with the largest component sum such that $\Phi \leq \Psi(\alpha)$. Using Lemma 4.5 we decompose $\Phi$ into short nonnegative loops $\Phi^{(1)}, \ldots, \Phi^{(k)}$, each with component sum at most $(1+(n+m) W)^{n+m}$. Since $\mu^{\tilde{\sigma}}(p) \geq W$ for all $p \in S$ and $\bullet t \in S$ for all $t \in \Phi^{(j)}$, $j \in[k]$, we can use Lemma 4.3 to find transition sequences $\tau^{(1)}, \ldots, \tau^{(k)}$ with $\Psi\left(\tau^{(j)}\right)=\Phi^{(j)}$, $j \in[k]$, such that $\tau:=\tau^{(1)} \cdots \tau^{(k)}$ is enabled at $\mu^{\tilde{\sigma}}$. Let $\mu^{\tilde{\sigma}} \stackrel{\tau}{\rightarrow} \mu^{\tau}$. For each $p \in S$, we observe $\Delta(\Phi)(p)<W$. To see this, assume $\Delta(\Phi)(p) \geq W$. By the maximality of $\Phi, \Psi(\alpha)-\Phi$ doesn't contain a transition $t$ with $p=\bullet t$. Therefore, $\Delta(\alpha)(p)=\Delta(\Phi)(p)+\Delta(\Psi(\alpha)-\Phi)(p) \geq W$. But then, $\mu^{\tilde{\sigma}}(p)+\Delta(\alpha)(p) \geq \ell_{\mathrm{big}}+W=\ell_{\text {fire }}$, a contradiction to the fact that no place of $S$ is firing. Since all $\tau^{(j)}$ are nonnegative loops, we obtain $\Delta\left(\tau^{(1)} \cdots \tau^{(j)}\right)(p) \leq W$ for all $p \in S$ and $j \in[k]$. Furthermore, $\left|\tau^{(j)}\right|<(1+(n+m) W)^{n+m}$ implies $\Delta\left(\tau_{(i)}^{(j)}\right)(p) \leq(1+(n+m) W)^{n+m} W$ for all $i \in\left[\left|\tau^{(j)}\right|\right]$ and $p \in P$. We obtain $\max \left(\mu^{\tilde{\sigma}}+\Delta\left(\tau^{(1)} \cdots \tau^{(j-1)}\right), \tau^{(j)}, S\right) \leq \ell_{b-1}+W+(1+(n+m) W)^{n+m} W \leq \ell_{b}$ for all $j \in[k]$, and thus our first important intermediate result of the proof:

$$
\max \left(\mu^{\tilde{\sigma}}, \tau, S\right) \leq \ell_{b}
$$

In other words, the token numbers of places of $S$ at all markings obtained while firing $\tau$ at $\mu^{\tilde{\sigma}}$ are at most $\ell_{b}$. 

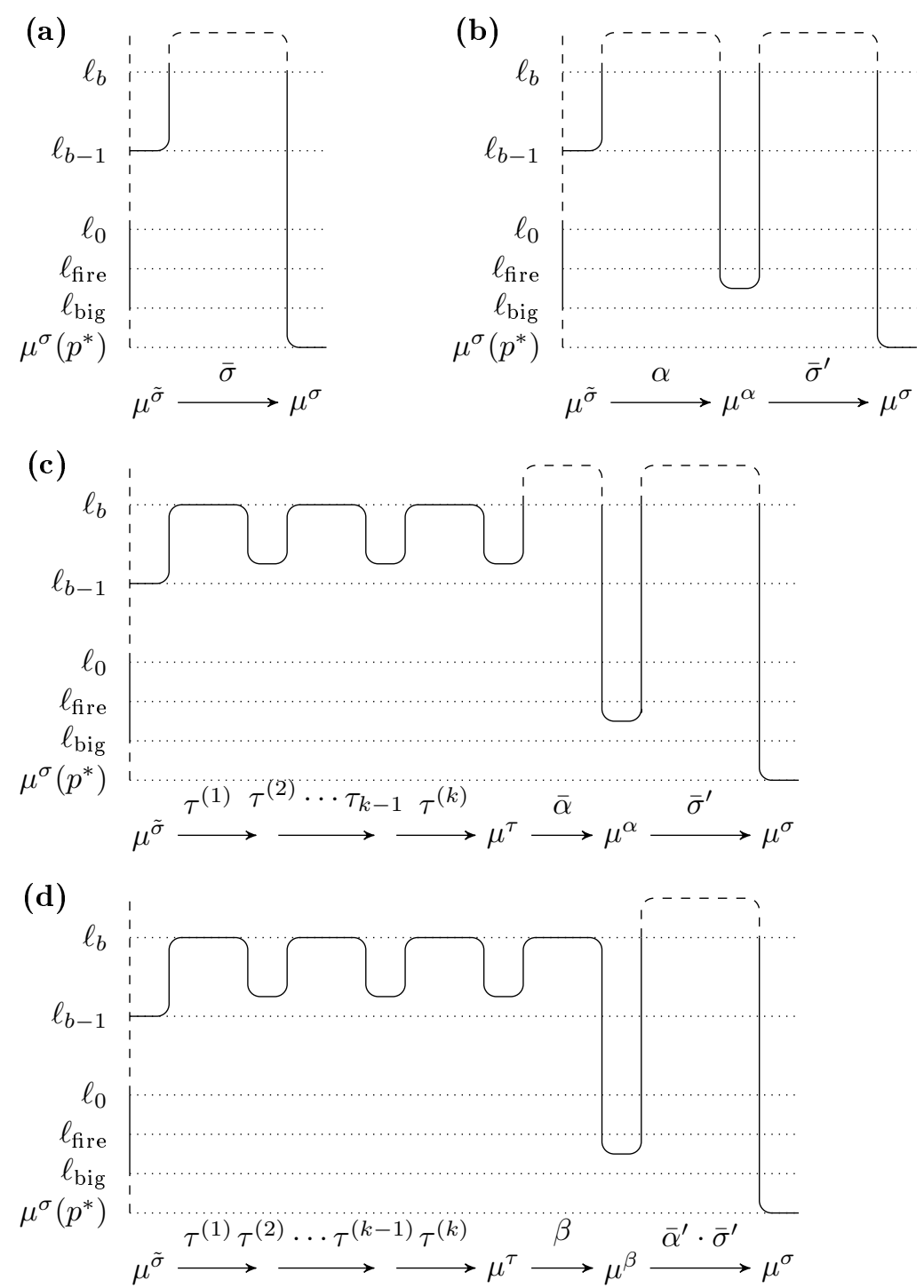

Figure 2: (a)-(d) illustrate the development of the number of tokens at a place $p^{*}$ which is big at $\mu^{\tilde{\sigma}}$ during certain steps of the permutation procedure described in Lemma 4.6. The number of tokens is bounded from above by the respective curve. The number of big places at $\mu^{\tilde{\sigma}}$ is $b$. Dashed lines symbolize that the number of tokens can become arbitrarily big.

We now consider $\Psi(\alpha)-\Phi$. Observing $\mu^{\tau}(p) \geq \mu^{\tilde{\sigma}}(p) \geq W$ and $\mu^{\sigma}(p) \geq W$ for all $p \in S$, and $\bullet \bar{\alpha} \subseteq S$ for some transition sequence $\bar{\alpha}$ with $\Psi(\bar{\alpha})=\Psi(\alpha)-\Phi$, we use Lemma 4.3 to find a transition sequence $\bar{\alpha}$ with $\Psi(\bar{\alpha})=\Psi(\alpha)-\Phi$ that is enabled at $\mu^{\tau}$, see (c) of Figure 2 .

We initialize another empty transition sequence $\beta \leftarrow()$, as well as $\bar{\alpha}^{\prime} \leftarrow \bar{\alpha}$. As long as there is a firing place of $S$ at $\mu^{\tau}+\Delta(\beta)$, we select a place $p \in S$ with $\max \left(\mu^{\tau}+\Delta(\beta), S\right)=\left(\mu^{\tau}+\Delta(\beta)\right)(p)$ and the transition $t \in \Psi_{\text {first }}\left(\bar{\alpha}^{\prime}\right)$ with $p=\bullet t$, and set $\beta \leftarrow \beta \cdot t$, as well as $\bar{\alpha}^{\prime} \leftarrow \bar{\alpha}^{\prime} \doteq t$. It is important to note the difference of this selection procedure compared to the one before. Here, we select a place of $S$ with the largest number of tokens. Also note that $\beta$ is nonempty since $\mu^{\tilde{\sigma}}$ has a firing place in $S$ and $\mu^{\tau} \geq \mu^{\tilde{\sigma}}$. Let $\mu^{\beta}:=\mu^{\tau}+\Delta(\beta)$. By Lemma 4.2 , we observe $\mu^{\tau} \stackrel{\beta}{\rightarrow} \mu^{\beta}$, and $\bar{\alpha}^{\prime}$ is enabled at $\mu^{\beta}$. In total, we have $\mu^{\tilde{\sigma}} \stackrel{\tau}{\rightarrow} \mu^{\tau} \stackrel{\beta}{\rightarrow} \mu^{\beta} \stackrel{\bar{\alpha}^{\prime} \cdot \bar{\sigma}^{\prime}}{\rightarrow} \mu^{\sigma}$.

We observe $\max \left(\mu^{\tau}, S\right)=\max \left(\mu^{\tilde{\sigma}}+\Delta(\tau), S\right) \leq \max \left(\mu^{\tilde{\sigma}}, S\right)+W \leq \ell_{b-1}+W$. Now, for 
the sake of contradiction, assume that $\max \left(\mu^{\tau}, \beta, S\right)>\ell_{b}$. Then, $\max \left(\mu^{\tau}+\Delta\left(\beta_{(i)}\right), S\right)>\ell_{b} \geq$ $\ell_{b-1}+W+2 n W \geq \max \left(\mu^{\tau}, S\right)+2 n W$ for some $i \in[|\beta|]$. But then, Lemma 4.4 implies that $\beta$ contains a positive loop, a contradiction to the maximality of $\Phi$. Therefore, $\max \left(\mu^{\tau}, \beta, S\right) \leq \ell_{b}$. We merge $\tau$ and $\beta$ and obtain the nonempty transition sequence $\gamma:=\tau \cdot \beta$.

Our observations can now be summarized as our second important intermediate result, also see (d) of Figure 2.

$$
\mu^{\tilde{\sigma}} \stackrel{\gamma}{\rightarrow} \mu^{\beta} \stackrel{\bar{\alpha}^{\prime} \cdot \bar{\sigma}^{\prime}}{\longrightarrow} \mu^{\sigma}, \quad|\gamma|>0, \quad \max \left(\mu^{\tilde{\sigma}}, \gamma, S\right) \leq \ell_{b}, \quad \text { and } \quad \max \left(\mu^{\beta}, S\right)<\ell_{\text {fire }} .
$$

As the last step, consider the smallest $j \in[|\gamma|]$ such that the number of big places at $\mu^{\tilde{\sigma}}+\Delta\left(\gamma_{(j)}\right)$ is at least $b+1$. If such a $j$ does not exist, set $j:=|\gamma|$. Now define $\tilde{\sigma}^{\text {new }}:=\tilde{\sigma} \cdot \gamma_{(j)}$, as well as $\bar{\sigma}^{\text {new }}:=\gamma_{(j+1,|\gamma|)} \cdot \bar{\alpha}^{\prime} \cdot \bar{\sigma}^{\prime}$. Observe that $\tilde{\sigma}^{\text {new }}$ is longer than $\tilde{\sigma}$, and, together with $\bar{\sigma}^{\text {new }}$, satisfies the invariants (i)-(iii). In particular, if there is still a big place at the end of the step, then every place that is big at some time during the step is also big at the end of it.

By iteratively applying this procedure, we obtain a permutation $\varphi$ of $\sigma$ such that $\mu^{(0)} \stackrel{\varphi}{\rightarrow}$ $\mu^{\sigma}$ and $\max \left(\mu^{(0)}, \varphi\right) \leq \ell_{n}$, i.e., all markings obtained while firing $\varphi$ contain at most $\ell_{n}$ tokens at each place. Note that if one of the values $n, m, W$ is 0 , then only the initial marking $\mu^{(0)}$ is reachable. Therefore, we can choose an appropriate constant $c$ such that $\ell_{n} \leq(2 n m W+$ $\left.\max \left(\mu^{(0)}\right)+\max (\mu)\right)^{c(n+m)}$ for all possible inputs as defined at the beginning.

We can use Lemma 4.6 to show that the reachability and the covering problems of gcf-PNs are PSPACE-complete.

Theorem 4.7. The zero-reachability, the reachability, and the covering problems of gcf-PNs are PSPACE-complete, even if restricted to gss-PNs.

Proof. The PSPACE-hardness of these problems is shown in Lemma 3.5. It remains to be shown that these problems are in PSPACE. Consider a gcf-PN $\mathcal{P}=\left(P, T, F, \mu^{(0)}\right)$, and a marking $\mu$ of $\mathcal{P}$. Assume $\mu$ is reachable in $\mathcal{P}$. Then, there is a firing sequence $\sigma$ with $\mu^{(0)} \stackrel{\sigma}{\rightarrow} \mu$. By Lemma 4.6 there is a permutation $\varphi$ of $\sigma$ with $\mu^{(0)} \stackrel{\varphi}{\rightarrow} \mu$ such that $\max \left(\mu^{(0)}, \varphi\right) \leq\left(2 n m W+\max \left(\mu^{(0)}\right)+\right.$ $\max (\mu))^{c(n+m)}$, i.e., the encoding size of each marking obtained while firing $\varphi$ is polynomial in $\operatorname{size}(\mathcal{P}, \mu)$. As before in the proof of Theorem 4.1 this implies that the problem is in PSPACE.

For the covering problem observe that $\mu$ can be covered in $\mathcal{P}=\left(P, T, F, \mu^{(0)}\right)$ if and only if $\mu$ is reachable in the wipe-extension $\mathcal{P}^{-}=\left(P, T^{-}, F^{-}, \mu^{(0)}\right)$ (which is a gcf-PN itself): If $\mu$ can be covered in $\mathcal{P}$, then there is a firing sequence $\sigma$ of $\mathcal{P}$ leading to a marking $\mu^{\prime} \geq \mu$. The same sequence is enabled in $\mathcal{P}^{-}$, and it can be extended by transitions of $T^{-} \backslash T$ such that the resulting firing sequence $\sigma^{\prime}$ leads to $\mu$. On the other hand, if $\mu$ is reachable in $\mathcal{P}^{-}$, then there is a firing sequence $\sigma$ of $\mathcal{P}^{-}$leading to $\mu$. By removing all occurrences of the new transitions in $T^{-} \backslash T$ from $\sigma$, we obtain a firing sequence $\sigma^{\prime}$ of $\mathcal{P}$ leading to a marking $\mu^{\prime} \geq \mu$.

\section{Canonical Firing Sequences, and the Boundedness, Con- tainment, and Equivalence Problems}

The canonical permutation obtained in Section 4 is, by itself, not strong enough to show the membership of the boundedness problem in PSPACE or to yield algorithms deciding the containment and equivalent problems. Therefore, our first objective in this section is to distill a strong form of canonical firing sequences from canonical permutations.

Lemma 5.1. There is a constant $c$ such that, for each reachable marking $\mu$ of a gcf-PN $\mathcal{P}=$ $\left(N, \mu^{(0)}\right)$, there are transition sequences $\xi, \bar{\xi}, \alpha^{(1)}, \ldots, \alpha^{(k)}, \tau^{(1)}, \ldots, \tau^{(k)}$ for some $k \leq n \cdot \max (\mu)$ having the following properties.

(a) $\xi=\alpha^{(1)} \cdot \tau^{(1)} \cdot \alpha^{(2)} \cdot \tau^{(2)} \cdots \alpha^{(k)} \cdot \tau^{(k)}$ is a firing sequence leading from $\mu^{(0)}$ to $\mu$.

(b) $\bar{\xi}=\alpha^{(1)} \cdot \alpha^{(2)} \cdots \alpha^{(k)}$ is a firing sequence with $|\bar{\xi}| \leq\left(2 n m W+\max \left(\mu^{(0)}\right)\right)^{c n(n+m)}$. 
(c) Each $\tau^{(i)}, i \in[k]$, is a positive loop with $\left|\tau^{(i)}\right| \leq\left(2 n m W+\max \left(\mu^{(0)}\right)\right)^{c n(n+m)}$ enabled at some marking $\mu^{*}$ with $\max \left(\mu^{*}\right) \leq\left(2 n m W+\max \left(\mu^{(0)}\right)\right)^{c(n+m)}$ and $\mu^{*} \leq \mu^{(0)}+\Delta\left(\alpha^{(1)} \cdot \alpha^{(2)} \cdots \alpha^{(i)}\right)$.

Proof. As before, we assume $n, m, W>0$ since otherwise the claim holds. Let $\mu$ be a reachable marking of a gcf-PN $\mathcal{P}=\left(P, T, F, \mu^{(0)}\right)$. Then, there is a firing sequence $\sigma$ leading to $\mu$. Consider the wipe-extension $\mathcal{P}^{-}=\left(P, T^{-}, F^{-}, \mu^{(0)}\right)$ of $\mathcal{P}$ having $n$ places, $m+n$ transitions, and largest edge multiplicity $W$. The sequence $\sigma$ is also a firing sequence in $\mathcal{P}^{-}$leading to $\mu$, and can be extended by transitions of $T^{-} \backslash T$ to a firing sequence $\sigma^{\prime}$ leading to the empty marking $\mu^{\mathrm{e}}$ having no tokens at all places. Using Lemma 4.6 we obtain a canonical permutation $\varphi$ of $\sigma^{\prime}$ such that $\max \left(\mu^{(0)}, \varphi\right) \leq$ $\left(2 n(m+n) W+\max \left(\mu^{(0)}\right)+\max \left(\mu^{\mathrm{e}}\right)\right)^{c(2 n+m)}=\left(2 n(m+n) W+\max \left(\mu^{(0)}\right)\right)^{c(2 n+m)}=: b$ in $\mathcal{P}^{-}$. Define the markings $\nu^{(i)}, i \in[0,|\varphi|]$, by $\mu^{(0)} \stackrel{\varphi(i)}{\longrightarrow} \nu^{(i)}$. Since $\max \left(\nu^{(i)}\right) \leq b$ for all $i$, each contiguous subsequence of $\left(\nu^{(0)}, \ldots, \nu^{(|\varphi|)}\right)$ with length at least $(b+1)^{n}$ contains some marking at least twice. Therefore, each such subsequence corresponds to a subsequence of $\varphi$ with displacement 0 at all places, a zero-loop. Furthermore, each such zero-loop is enabled at some marking with at most $b$ tokens at each place.

We split $\varphi$ into contiguous nonoverlapping subsequences $\varphi^{(1)}, \ldots, \varphi^{(\ell)}$ with $\varphi=\varphi^{(1)} \cdots \varphi^{(\ell)}$ such that, for each $\nu^{(i)}, i \in[0,|\varphi|]$, there is exactly one $j \in[\ell]$ with $\mu^{(0)} \stackrel{\varphi^{(1)} \cdots \varphi^{(j)}}{\longrightarrow} \nu^{(i)}$ in $\mathcal{P}^{-}$, i.e., each marking $\nu^{(i)}$ which could potentially enable a zero-loop, is witnessed by the sequences $\varphi^{(1)}, \ldots, \varphi^{(\ell)}$. Note that $\ell \leq(b+1)^{n}$. Define $\eta^{(j)}, j \in[\ell]$, by $\mu^{(0)} \stackrel{\varphi^{(1)} \ldots \varphi^{(j)}}{\longrightarrow} \eta^{(j)}$ in $\mathcal{P}^{-}$. Now, as long as there is a sequence $\varphi^{(j)}, j \in[\ell]$, with length at least $(b+1)^{n}$, we find a zero-loop $\vartheta$ of length at most $(b+1)^{n}$ within $\varphi^{(j)}$, remove $\vartheta$ from $\varphi^{(j)}$, and add it to a list $L$. Notice that after this procedure, each $\varphi^{(j)}, j \in[\ell]$, has length less than $(b+1)^{n}$, and each $\nu^{(i)}, i \in[0,|\varphi|]$, is still witnessed by the sequences $\varphi^{(1)}, \ldots, \varphi^{(\ell)}$ since we only removed zero-loops, i.e., we still have $\mu^{(0)} \stackrel{\varphi^{(1)} \cdots \varphi^{(j)}}{\longrightarrow} \eta^{(j)}$. In particular, the sequences $\varphi^{(1)} \cdots \varphi^{(\ell)}$ and $\varphi^{(1)} \cdot \vartheta^{(1)} \cdots \vartheta^{\left(i_{1}\right)} \cdot \varphi^{(2)}$. $\vartheta^{\left(i_{1}+1\right)} \cdots \vartheta^{\left(i_{2}\right)} \cdot \varphi^{(3)} \cdots \varphi^{(k)} \cdot \vartheta^{\left(i_{\ell}+1\right)} \cdots \vartheta^{\left(i_{\ell+1}\right)}$ are enabled at $\mu^{(0)}$ in $\mathcal{P}^{-}$where the sequences $\vartheta^{(i)}$, $i \in\left[1, i_{\ell+1}\right]$, are the conveniently numbered zero-loops of $L$.

Now, let $\beta^{(j)}, j \in[\ell]$, be the sequence obtained by removing all transitions of $T^{-} \backslash T$ from $\varphi^{(j)}$. Equivalently, we obtain $\gamma^{(i)}, i \in\left[1, i_{\ell+1}\right]$, from $\vartheta^{(i)}$. First note that $\beta^{(1)} \cdots \beta^{(\ell)}$ is a firing sequence of $\mathcal{P}$ with total length at most $(b+1)^{2 n}$ since there are at most $(b+1)^{n}$ markings to be witnessed and each sequence $\varphi^{(j)}$, and therefore $\beta^{(j)}$, has length at most $(b+1)^{n}$. Let $\mu^{(0)} \stackrel{\beta^{(1)} \ldots \beta^{(j)}}{\longrightarrow} \mu^{(j)}$ in $\mathcal{P}$. Next, notice that the transition $t_{i}^{-} \in T^{-} \backslash T, i \in[n]$, was removed from the sequences exactly $\mu\left(p_{i}\right)$ times. Therefore, at $\operatorname{most} n \cdot \max (\mu)$ of the sequences $\gamma^{(i)}$ are positive loops (instead of zero-loops). We only keep the loops that are positive loops, and call them $\tau^{(1)}, \ldots, \tau^{(k)}$ where $k \leq n \cdot \max (\mu)$. Each of these positive loops is enabled at some marking $\nu^{(i)}, i \in[0,|\varphi|]$, and for each $\nu^{(i)}$ there is a $j$ such that $\nu^{(i)}=\eta^{(j)} \leq \mu^{(j)}$. This means that each positive loop $\tau^{(i)}$ is enabled at some marking $\mu^{(j)}$. Therefore, we can find $k$ transition sequences $\alpha^{(1)}, \ldots, \alpha^{(k)}$ with $\alpha^{(1)} \cdots \alpha^{(k)}=\beta^{(1)} \ldots \beta^{(\ell)}$ such that the sequences $\alpha^{(i)}, \tau^{(i)}, i \in[k]$, satisfy the properties of the lemma, where we assume w.l.o.g. that the positive loops are conveniently numbered.

We call the sequence $\bar{\xi}$ the backbone of the canonical sequence under consideration. Using canonical firing sequences as constructed in Lemma 5.1. we can show the following lemma.

Lemma 5.2. There is a constant $c$ such that, for each gcf-PNP $=\left(P, T, F, \mu^{(0)}\right), \mathcal{P}$ is unbounded if and only if there is a reachable marking $\mu$ with $\max (\mu) \geq \max \left(\mu^{(0)}\right)+\delta+1$ if and only if there is a reachable marking $\mu$ with $\max (\mu) \in\left[\max \left(\mu^{(0)}\right)+\delta+1, \max \left(\mu^{(0)}\right)+2 \delta+1\right]$ where $\delta=\left(2 n m W+\max \left(\mu^{(0)}\right)\right)^{c n(n+m)} \cdot W$.

Proof. As before, we assume $n, m, W>0$ since otherwise only $\mu^{(0)}$ is reachable and the lemma holds. If $\mathcal{P}$ is unbounded, then there is a reachable marking $\bar{\mu}$ with $\max (\bar{\mu}) \geq \max \left(\mu^{(0)}\right)+\delta+1$. If such a marking $\bar{\mu}$ exists, then $\delta>W$ implies that one of the markings $\mu$ observed while firing a firing sequence leading to $\bar{\mu}$ satisfies $\max (\mu) \in\left[\max \left(\mu^{(0)}\right)+\delta+1, \max \left(\mu^{(0)}\right)+2 \delta+1\right]$. If such a marking $\mu$ exists, then the canonical firing sequence $\xi$ of Lemma 5.1 leading to $\mu$ contains a loop $\tau^{(i)}$ with $\Delta\left(\tau^{(i)}\right)(p)>0$ for some place $p$ with $\mu(p)=\max (\mu)$ since $\Delta(\bar{\xi}) \leq \boldsymbol{\delta}$, implying the unboundedness of $\mathcal{P}$. 
We can now prove the following theorem.

Theorem 5.3. The boundedness problem of gcf-PNs is PSPACE-complete, even if restricted to gss-PNs.

Proof. Since the PSPACE-hardness was shown in Lemma 3.5, it remains to be shown that it is in PSPACE. By Lemma 5.2, we have to check if a reachable marking $\mu$ as defined in the lemma exists. Hence, in order to check if $\mathcal{P}$ is unbounded, we guess $\mu$ in polynomial time, and check in polynomial space if $\mu$ is reachable by using Theorem 4.7. As before, this nondeterministic algorithm implies a deterministic polynomial space algorithm.

In the following, we show a doubly exponential space upper bound for the containment and the equivalence problems. Let $\mathcal{P}$ and $\mathcal{P}^{\prime}$ be the gcf-PNs of interest. The proof idea for the upper bound is as follows. First we show, how to compute semilinear representations of $\mathcal{R}(\mathcal{P})$ and $\mathcal{R}\left(\mathcal{P}^{\prime}\right)$ s.t. each representation has doubly exponential size in the combined size of $\mathcal{P}$ and $\mathcal{P}^{\prime}$. Then, we simply apply known bounds for the equivalence, and the containment problems of semilinear sets. The representation of $\mathcal{R}(\mathcal{P})$ is computed as follows. We consider all possible backbones of canonical firing sequences of $\mathcal{P}$. Each of these backbones $\bar{\xi}$ constitutes its own linear set, where the constant vector is the marking reached by the backbone, and the set of periods is the set of the displacements of all short positive loops enabled at some marking obtained while firing the backbone.

Lemma 5.4. Given a gcf-PN $\mathcal{P}=\left(P, T, F, \mu^{(0)}\right)$, we can construct a semilinear representation of $\mathcal{R}(\mathcal{P})$ in doubly exponential time in $\operatorname{size}(\mathcal{P})$.

Proof. For convenience, let $u:=\left(2 n m W+\max \left(\mu^{(0)}\right)\right)^{c(n+m)}$ where $c$ is the constant of Lemma 5.1. Let $\Sigma_{\ell}$ denote the set of all firing sequences of length $\ell$. For a firing sequence $\sigma$, the set $M_{\sigma}^{*}$ contains all markings $\mu^{*}$ with $\max \left(\mu^{*}\right) \leq u$ and $\mu^{*} \leq \mu^{(0)}+\Delta\left(\sigma_{(i)}\right)$ for some $i \in[0,|\sigma|]$. For a marking $\mu^{*}$, the set $D_{\mu^{*}}$ contains the displacements of all positive loops $\tau$ enabled at $\mu^{*}$ with $\Delta(\tau)(p) \leq u^{n} W$ for all $p \in P$. Consider the set

$$
S L:=\bigcup_{\ell=0}^{\left\lfloor u^{n}\right\rfloor} \bigcup_{\bar{\xi} \in \Sigma_{\ell}} L\left(\mu^{(0)}+\Delta(\bar{\xi}), \bigcup_{\mu^{*} \in M_{\bar{\xi}}^{*}} D_{\mu^{*}}\right) .
$$

Similar to the backbone of a canonical sequence of Lemma 5.1, we call each sequence of all $\Sigma_{\ell}$ backbone.

To see $S L \subseteq \mathcal{R}(\mathcal{P})$, notice that, by construction of $S L$, a marking in $S L$ can be reached by a firing sequence which results from inserting positive loops $\tau$ with displacement at most $u^{n} W$ at all places into a backbone $\bar{\xi}$ of length at most $\left\lfloor u^{n}\right\rfloor$ at appropriate positions which enable $\tau$. Now we show $\mathcal{R}(\mathcal{P}) \subseteq S L$. Let $\mu \in \mathcal{R}(\mathcal{P})$ be a reachable marking. Consider the sequences $\xi, \bar{\xi}, \tau^{(1)}, \ldots, \tau^{(k)}$ of Lemma 5.1 where $\mu^{(0)} \stackrel{\xi}{\rightarrow} \mu$. Then, $\Delta\left(\tau^{(i)}\right) \in \bigcup_{\mu^{*} \in M_{\bar{\xi}}^{*}} D_{\mu^{*}}$ for all $i \in[k]$ proving $\mu \in S L$.

It remains to be shown that $S L$ can be constructed in doubly exponential time. We first construct all relevant sets $D_{\mu^{*}}$. To this end, consider all marking $\mu^{*}$ with $\max \left(\mu^{*}\right) \leq u$ one after another. For each such $\mu^{*}$, we check for all markings $\mu$ with $\mu^{*}<\mu$ and $\mu(p) \leq \mu^{*}(p)+u^{n} W$ for all places $p \in P$ if $\mu$ can be reached from $\mu^{*}$. If this is the case for some $\mu, \mu-\mu^{*}$ is the displacement of a positive loop enabled at $\mu^{*}$, and we add $\mu-\mu^{*}$ to $D_{\mu^{*}}$. By Theorem 4.7 this can be done in polynomial space in $\operatorname{size}(\mathcal{P})$ since the encoding sizes of all $\mu^{*}$ and $\mu$ under consideration are polynomially bounded by $\operatorname{size}(\mathcal{P})$ (i.e., $\operatorname{size}\left(\left(P, T, F, \mu^{*}\right), \mu\right) \leq p(\operatorname{size}(\mathcal{P}))$ for some polynomial $\left.p\right)$. Since there are at most exponentially many possibilities for $\mu^{*}$ and $\mu$, respectively, the running time to compute all sets $D_{\mu^{*}}$ is also at most exponential.

Now, we generate all backbones $\bar{\xi}$ of length at most $u^{n}$ one after another. Each generated sequence corresponds to a linear set. Since $\left|\Sigma_{\ell}\right| \leq m^{\ell} \leq m^{u^{n}}$, the total number of produced linear sets is at most doubly exponential in $\operatorname{size}(\mathcal{P})$. When generating the linear set corresponding to a backbone $\bar{\xi}$, we fire the transitions one by one and compare the current marking $\mu$ with all possible $\mu^{*}$ under consideration. If $\mu^{*} \leq \mu$, we add all elements of $D_{\mu^{*}}$ to our set of periods. Therefore, this procedure needs at most doubly exponential time in $\operatorname{size}(\mathcal{P})$ to compute $S L$. 
Theorem 5.5. The containment and the equivalence problems of gcf-PNs are PSPACE-hard and decidable in doubly exponential space, even if restricted to gss-PNs.

Proof. We first show PSPACE-hardness for these problems. Let $\mathcal{P}=\left(P, T, F, \mu^{(0)}\right)$ be a gss-PN, and $\delta$ be defined as in Lemma 5.2 . We construct the gss-PN $\mathcal{P}^{+}=\left(P, T^{+}, F^{+}, \mu^{(0)}\right)$ from $\mathcal{P}$ by adding, for all places $p_{i}, p_{j} \in P$, a new transition $t_{i, j}$ with $F^{+}\left(p_{i}, t_{i, j}\right)=\max \left(\mu^{(0)}\right)+\delta+1$ and $F^{+}\left(t_{i, j}, p_{j}\right)=\max \left(\mu^{(0)}\right)+\delta+2$. Notice that for each $k \in \mathbb{N}$, the net $\mathcal{P}^{+}$has a reachable marking $\mu$ with $\mu(p) \geq k$ for all $p \in P$ if and only if $\mathcal{P}$ is unbounded. Now, consider the wipe-extension $\mathcal{P}^{+-}$ of $\mathcal{P}^{+}$which is also a gss-PN. In $\mathcal{P}^{+-}$, every marking is reachable if and only if $\mathcal{P}$ is unbounded. Let $\mathcal{P}^{\prime}=\left(P, T^{\prime}, F^{\prime}, \nu^{(0)}\right)$ be the gss-PN where $\nu^{(0)}$ is the empty marking, and for each place $p \in P, \mathcal{P}^{\prime}$ has a transition $t \in T^{\prime}$ with $F^{\prime}(t, p)=1$. Observe that every marking of $\mathcal{P}^{\prime}$ is reachable. Therefore, $\mathcal{P}$ is unbounded if and only if $\mathcal{R}\left(\mathcal{P}^{\prime}\right)=\mathcal{R}\left(\mathcal{P}^{+-}\right)$if and only if $\mathcal{R}\left(\mathcal{P}^{\prime}\right) \subseteq \mathcal{R}\left(\mathcal{P}^{+-}\right)$, proving the PSPACE-hardness of the equivalence and the containment problems of gss-PNs.

It remains to be shown that the containment and the equivalence problems are decidable in doubly exponential space. Let $\mathcal{P}_{1}$ and $\mathcal{P}_{2}$ be two gcf-PNs. Using Lemma 5.4 we compute semilinear representations $S L_{1}$ and $S L_{2}$ of $\mathcal{R}\left(\mathcal{P}_{1}\right)$ and $\mathcal{R}\left(\mathcal{P}_{2}\right)$ in doubly exponential time, respectively. By [10] (also see [12] for a simpler proof), the equivalence problem of semilinear sets is in $\Pi_{2}^{p}$, the complement of the second level of the polynomial hierarchy. Therefore, the equivalence of $S L_{1}$ and $S L_{2}$ can be verified in polynomial space in the size of $S L_{1}$ and $S L_{2}$, i.e., in doubly exponential space in the combined encoding size of $\mathcal{P}_{1}$ and $\mathcal{P}_{2}$. For the containment problem note that $\mathcal{R}\left(\mathcal{P}_{1}\right) \subseteq \mathcal{R}\left(\mathcal{P}_{2}\right)$ if and only if $\mathcal{R}\left(\mathcal{P}_{1}\right) \cup \mathcal{R}\left(\mathcal{P}_{2}\right)=\mathcal{R}\left(\mathcal{P}_{2}\right)$ if and only if $S L^{\prime}=S L_{2}$ where $S L^{\prime}$ is a semilinear representation of $\mathcal{R}\left(\mathcal{P}_{1}\right) \cup \mathcal{R}\left(\mathcal{P}_{2}\right)$ which can easily be be obtained by combining $S L_{1}$ and $S L_{2}$.

Our construction is similar to that given in [16] for cf-PNs which uses results of [23, and yields a semilinear representation of the reachability set of cf-PNs having single exponential encoding size, implying single exponential space algorithms for the containment and equivalence problems. The difference in the encoding sizes of these semilinear representation between cf-PNs and gcf-PNs does not result from the slight differences in the canonical firing sequences themselves (in fact, our canonical sequence can also be used to generate the semilinear representations for cf-PNs in single exponential time), rather, it results from the following.

For cf-PNs, we used that each nonnegative loop that is intermediately enabled by some backbone can be partitioned into suitable nonnegative loops which are intermediately enabled by every other backbone with the same Parikh image. Therefore, it is sufficient to only consider one of these backbones. This results in a single exponential number of relevant backbones, and therefore in a single exponential number of linear sets, each of single exponential size. However, the same strategy fails in the case of gcf-PNs since the order of the transitions is much more relevant for gcf-PNs than for cf-PNs: firing transitions in a certain order can intermediately enable loops that cannot be partitioned further and that are not intermediately enabled by firing the same transitions in some other order. This is illustrated in Figure 3. Hence, to improve the doubly exponential space bound for the equivalence problem, some other or a refined approach will have to be found.

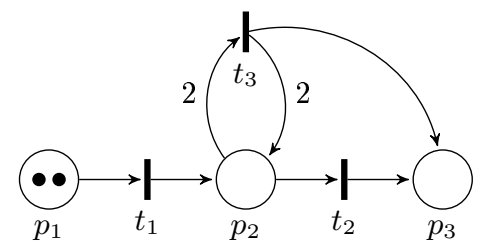

Figure 3: The firing sequences $t_{1} t_{1} t_{2} t_{2}$ and $t_{1} t_{2} t_{1} t_{2}$ have the same Parikh image but only the first sequence intermediately enables the positive loop $t_{3}$. 


\section{Exponent-sensitive Commutative Grammars}

For the definitions of commutative grammars and context-free commutative grammars (cfc grammars) in particular, we refer to [11. Based on our results for gcf-PNs, we can give complexity results for the uniform word problem of a new class of commutative grammars we call exponentsensitive commutative grammars (esc grammars). An esc grammar is a quadruple $(N, T, S, P)$, where $N$ is a finite set of variables (i.e., nonterminal symbols), $T$ with $T \cap N=\emptyset$ is a finite set of terminal symbols, $S \in N$ is the start symbol (i.e., the axiom), and $P \subset\left\{\{x\}^{\oplus} \mid x \in N\right\} \times(N \cup T)^{\odot}$ is a finite set of productions. Here, $M^{\odot}$ and $M^{\oplus}$ denote the free commutative monoid and the free commutative semigroup on a set $M$, respectively. In other words, productions of an esc grammar can substitute many occurences of a single variable $x \in N$ at once by variables and terminal symbols while productions of cfc grammars can substitute only one occurrence of a single variable. Hence, in a certain sense, we have given up a little bit of context-freeness. Note, though, that the families of esc grammars and of context-sensitive commutative grammars (csc grammars) are incomparable since esc grammars allow productions where the left-hand side of a production is longer than the right-hand side.

The uniform word problem of commutative grammars asks if, given a commutative grammar and a commutative word of $T^{\odot}$, if the word can be obtained from the start symbol by iteratively applying productions. This problem has the same complexity for esc grammars as the reachability problem of gcf-PNs.

Theorem 6.1. The uniform word problem of esc grammars is PSPACE-complete.

Proof. By identifying variables and terminal symbols by places of a gcf-PN, we can reduce the uniform word problem of esc grammars in logspace to the reachability problem of gcf-PNs.

On the other hand, we can reduce the reachability problem to the uniform word problem: Let $\mathcal{P}=\left(P, T, F, \mu^{(0)}\right)$ be a gcf-PN and $\mu$ a marking, where w.l.o.g. $P=\left\{p_{1}, \ldots, p_{n}\right\}$, and $T=\left\{t_{1}, \ldots, t_{m}\right\}$. We obtain the gcf-PN $\mathcal{P}^{\prime}=\left(P^{\prime}, T^{\prime}, F^{\prime}, \nu^{(0)}\right)$ from $\mathcal{P}$ as follows. We add a place $p_{n+1}$, such that $F^{\prime}\left(p_{n+1}, t_{i}\right)=F^{\prime}\left(t_{i}, p_{n+1}\right)=1$ for all $t_{i} \in T$ with $\bullet_{i}=\emptyset$, i.e., in the modified net, each transition has exactly one incoming edge. Next, we add, for each place $p_{i} \in P \cup\left\{p_{n+1}\right\}$, a new place $p_{i}^{*}$ and a transition $t_{i}^{*}$ with $F^{\prime}\left(p_{i}, t_{i}^{*}\right)=F^{\prime}\left(t_{i}^{*}, p_{i}^{*}\right)=1$. Then, we add a place $s$, and a transition $t_{s}$ with $F^{\prime}\left(s, t_{s}\right)=F\left(t_{s}, p_{n+1}\right)=1$ and $F^{\prime}\left(t_{s}, p_{i}\right)=\mu_{i}$ for all $p_{i} \in P$. Last, we define the marking $\nu^{(0)}$ by $\nu^{(0)}(s)=1$ and $\nu^{(0)}\left(p_{i}\right)=0$ for all places $p \neq s$, and the marking $\nu$ by $\nu(s)=0$, $\nu\left(p_{n+1}^{*}\right)=1$, and $\nu\left(p_{i}^{*}\right)=\mu\left(p_{i}\right)$ and $\nu\left(p_{i}\right)=\nu\left(p_{n+1}\right)=0$ for all $p_{i} \in P$. Note that $\mu$ is reachable in $\mathcal{P}$ if and only if $\nu$ is reachable in $\mathcal{P}^{\prime}$.

Now, we identify all places in $P \cup\left\{p_{n+1}, s\right\}$ with variables, $s$ with the start symbol, and all places $p_{i}^{*}, i \in[n+1]$ with terminal symbols. $\nu$ is mapped to the corresponding commutative word $w$, and each transition in the intuitive way to a production. Let $G$ be the resulting esc grammar. Then, $\nu$ is reachable in $\mathcal{P}^{\prime}$ if and only if $w$ can be produced by $G$. Therefore, the reachability problem of gcf-PNs and the uniform word problem of esc grammars have the same complexity which, by Theorem 4.7 is PSPACE-completeness.

Table 1 summarizes the complexities for the (uniform) word problem of different classes of grammars that are of particular interest in context of this paper.

\begin{tabular}{|l|l|l|l|}
\hline \multicolumn{2}{|c|}{ Class } & (Uniform) word problem & Reference \\
\hline \multirow{2}{*}{ Context-free } & non-commutative & P-complete & {$[3,13,[14,21,24$} \\
\cline { 2 - 4 } & commutative & NP-complete & {$[\underline{41}, 1]$} \\
\hline \multirow{2}{*}{ Context-sensitive } & non-commutative & PSPACE-complete & {$[15]$} \\
\cline { 2 - 4 } & commutative & PSPACE-complete & {$[11]$} \\
\hline Exponent-sensitive & commutative & PSPACE-complete & This paper \\
\hline Semi-groups & commutative & EXPSPACE-complete & {$[18]$} \\
\hline
\end{tabular}

Table 1: The complexities of some (uniform) word problems 


\section{References}

[1] S. Christensen. Distributed bisimularity is decidable for a class of infinite state-space systems. In W. Cleaveland, editor, CONCUR '92, volume 630 of Lecture Notes in Computer Science, pages 148-161. Springer Berlin / Heidelberg, 1992.

[2] S. Christensen, Y. Hirshfeld, and F. Moller. Bisimulation equivalence is decidable for basic parallel processes. In E. Best, editor, CONCUR'93, volume 715 of Lecture Notes in Computer Science, pages 143-157. Springer Berlin / Heidelberg, 1993.

[3] J. Cocke. Programming languages and their compilers: Preliminary notes. Courant Institute of Mathematical Sciences, New York University, 1969.

[4] J. Esparza. Petri nets, commutative context-free grammars, and basic parallel processes. Fundamenta Informaticae, 31(1):13-25, 1997.

[5] J. Esparza and M. Nielsen. Decibility issues for Petri nets - a survey. Journal of Informatik Processing and Cybernetics, 30(3):143-160, 1994.

[6] L. M. Ha, P. V. Trung, and P. T. H. Duong. A polynomial-time algorithm for reachability problem of a subclass of Petri net and chip firing games. In Computing and Communication Technologies, Research, Innovation, and Vision for the Future (RIVF), 2012 IEEE RIVF International Conference on, pages 1-6, 2012.

[7] R. R. Howell, P. Jancar, and L. E. Rosier. Completeness results for single-path Petri nets. Information and Computation, 106(2):253-265, 1993.

[8] R. R. Howell and L. E. Rosier. Completeness results for conflict-free vector replacement systems. Journal of Computer and System Sciences, 37(3):349-366, 1988.

[9] R. R. Howell, L. E. Rosier, and H.-C. Yen. Normal and sinkless Petri nets. In J. Csirik, J. Demetrovics, and F. Gécseg, editors, Fundamentals of Computation Theory, volume 380 of Lecture Notes in Computer Science, pages 234-243. Springer Berlin / Heidelberg, 1989.

[10] D. T. Huynh. The complexity of semilinear sets. In J. de Bakker and J. van Leeuwen, editors, Automata, Languages and Programming, volume 85 of Lecture Notes in Computer Science, pages 324-337. Springer Berlin / Heidelberg, 1980.

[11] D. T. Huynh. Commutative grammars: The complexity of uniform word problems. Information and Control, 57(1):21-39, 1983.

[12] D. T. Huynh. A simple proof for the sum upper bound of the inequivalence problem for semilinear sets. Elektronische Informationsverarbeitung und Kybernetik, pages 147-156, 1986.

[13] N. D. Jones and W. T. Laaser. Complete problems for deterministic polynomial time. Theoretical Computer Science, 3(1):105-117, 1976.

[14] T. Kasami. An efficient recognition and syntax-analysis algorithm for context-free languages. Technical report, Air Force Cambridge Research Lab, Bedford, MA, 1965.

[15] S.-Y. Kuroda. Classes of languages and linear-bounded automata. Information and Control, $7(2): 207-223,1964$.

[16] E. Mayr and J. Weihmann. Results on equivalence, boundedness, liveness, and covering problems of BPP-Petri nets. In J.-M. Colom and J. Desel, editors, Application and Theory of Petri Nets and Concurrency, volume 7927 of Lecture Notes in Computer Science, pages 70-89. Springer Berlin Heidelberg, 2013. 
[17] E. W. Mayr. An algorithm for the general Petri net reachability problem. In Proceedings of the thirteenth annual ACM symposium on Theory of computing, STOC '81, pages 238-246, New York, NY, USA, 1981. ACM.

[18] E. W. Mayr and A. R. Meyer. The complexity of the word problems for commutative semigroups and polynomial ideals. Advances in Mathematics, 46(3):305-329, 1982.

[19] L. Pottier. Minimal solutions of linear diophantine systems : bounds and algorithms. In R. Book, editor, Rewriting Techniques and Applications, volume 488 of Lecture Notes in Computer Science, pages 162-173. Springer Berlin / Heidelberg, 1991.

[20] W. J. Savitch. Relationships between nondeterministic and deterministic tape complexities. Journal of Computer and System Sciences, 4(2):177-192, 1970.

[21] M. Sipser. Introduction to the theory of computation. PWS Publishing Company, 1997.

[22] S. Taoka and T. Watanabe. Time complexity analysis of the legal firing sequence problem of Petri nets with inhibitor arcs. IEICE Trans. Fundam. Electron. Commun. Comput. Sci., E89-A:3216-3226, November 2006.

[23] H.-C. Yen. On reachability equivalence for BPP-nets. Theoretical Computer Science, 179(12):301-317, 1997.

[24] D. H. Younger. Recognition and parsing of context-free languages in time $n^{3}$. Information and Control, 10(2):189-208, 1967. 